\title{
Bending and Vibration Analysis of Curved FG Nanobeams via Nonlocal Timoshenko Model
}

\author{
S. A. H. Hosseini ${ }^{1}$, O. Rahmani* \\ ${ }^{1}$ Smart Structures and New Advanced Materials Laboratory, Department of Mechanical Engineering, University of Zanjan, Zanjan, Iran. \\ Corresponding author at: Department of Mechanical Engineering, University of Zanjan, Zanjan, Iran. E-mail address: omid.rahmani@znu.ac.ir \\ (Omid Rahmani), Tel.: +989122414371; Fax: +982432283204
}

\begin{abstract}
The bending and vibration behavior of a curved FG nanobeam using the nonlocal Timoshenko beam theory is analyzed in this paper. It is assumed that the material properties vary through the radius direction. The governing equations were obtained using Hamilton principle based on the nonlocal Timoshenko model of curved beam. An analytical approach for a simply supported boundary condition is conducted to analyze the vibration and bending of curved FG nanobeam. In the both mentioned analysis, the effect of significant parameter such as opening angle, the power law index of FGM, nonlocal parameter, aspect ratio and mode number are studied. The accuracy of the solution is examined by comparing the results obtained with the analytical and numerical results published in the literatures.
\end{abstract}

Keywords: Curved nanobeam; Vibration and bending; Nonlocal elasticity theory; Analytical solution; Functionally graded curved beam.

\section{Introduction}

Nowadays various fields of science and industry namely electronics, optics, aerospace, mechanical and civil engineering are considerably affected by nanotechnology ${ }^{[1,2]}$. To precisely design these structures having the knowledge of mechanical properties of nanostructure is strongly needed. For this purpose, numerous investigations are conducted to propose theories and methods to study the mechanical properties of such structures. Nonlocal theory that was proposed by Eringen is one of the best and commonest approaches used by researchers' community ${ }^{[3-21]}$. Based on this theory the stress at a reference point is a function of the strain at all neighbor points in the body. The natural frequency of nanotubes with consideration of surface effects using the nonlocal Timoshenko beam theory is studied in Lee $e t$ al. investigation ${ }^{[22]}$. In another study Wang et al. analyzed the free vibration of micro/nanobeams based on Eringen's nonlocal elasticity theory and Timoshenko beam theory ${ }^{[23]}$.

Currently nanocomposites are widely utilized in science and industry but because of their distinct interface which is accompanied with sudden variation in material properties they tend to lose their reliability ${ }^{[10]}$. To solve this phenomenon, there is a need to introduce a material, which has a smooth variation in its material properties. Functionally Graded Materials (FGM) have this characteristic which has made them to be largely used in various shape in numerous industries ${ }^{[2,24,25]}$. The free vibration of piezoelectric FG beams with rectangular cross sections in pre/post-buckling regimes is analyzed by Komijani et al. ${ }^{[26]}$ based on Timoshenko beam theory. Elather et al. analyzed the static- buckling behavior of functionally graded nanobeams as a core structure of micro and nano electro mechanical systems. The principle of virtual displacement is deployed to obtain the equilibrium equations. It was concluded that parameters such as material gradient index, boundary conditions and nonlocal effect have considerable influence on the static- buckling behavior of FG nanobeam ${ }^{[27]}$. They studied the static and buckling behavior of nonlocal FG Timoshenko nanobeam in their other investigation which showed that the material distribution profile influences the buckling and 
bending behavior of FG nanobeams ${ }^{[28]}$. Rahmani et al. used nonlocal beam theory to derive a closed-form solution for Timoshenko FG nanobeam to analyze the size effect on vibration behavior of the functionally graded nanobeam ${ }^{[24]}$. It was concluded that parameters such as the gradient index, length scale parameter and length-to-thickness ratio have crucial effect in investigation of free vibration of FG nanobeam. Simsek et al. examined the static bending and buckling of a FG nanobeam according to the nonlocal Timoshenko and Euler-Bernoulli beam theory. They concluded that their new proposed model produces larger deflection and smaller buckling load than the classical (local) beam model ${ }^{[29]}$.

Due to the crucial role of curved micro/nanobeam in designing the engineering structures, various investigations are conducted to study the mechanical behavior of these structures ${ }^{[30]}$. Farshi et al. analyzed the dynamic behavior of curved nanobeam considering the small scale effect. Their results showed a considerable difference between the results derived from their proposed model and those of classical theories ${ }^{[31]}$. Wang et al. analyzed the free vibration of nanorings/arches using the Eringen nonlocal theory with consideration of the small scale effect ${ }^{[32]}$.In another study made by Madina et al. the asymmetric buckling of a shallow initially curved microbeam subjected to distributed nonlinear deflection-dependent electrostatic forced was analyzed ${ }^{[33]}$.

Due to the previous literature review, there is no investigation that analyzed bending and vibration of FG curved nanobeam and almost all of the investigations were about straight nanobeams. Hence, the purpose of this paper is filling this gap. In this paper, the nonlocal Timoshenko beam theory is used to analyze the free vibration and bending of the curved FG nanobeams. Material properties are assumed to change through thickness direction. Using the Timoshenko and nonlocal theory of curved beam, the governing equations were derived. To analyze the vibration and bending of curved FG nanobeam an analytical approach for a simply supported boundary condition is employed. The effects of parameters such as the opening angle, the power law index of FGM, nonlocal parameter, aspect ratio and mode number on the vibration and bending of curved nanobeam is studied. Due to the negligible differences between the results of this paper and those of previous literature, it was concluded that our results are in excellent agreement with previous studies.

\section{Functionally Graded Materials properties}

It is assumed that the curved nanobeam is a mixture of steel and alumina. According to present method, the gradient variation may be chosen arbitrarily, though to simplify the mathematical treatment, it is common for them to be shown as exponential-type dependence. However, from an experimental point, it seems constructive to employ a power law gradient. Therefore, the effective material properties of the curved nanobeam are obtained by:

$$
\begin{aligned}
& E_{f}(z)=\left(E_{c}-E_{m}\right)\left(\frac{z}{h}+\frac{1}{2}\right)^{p}+E_{m} \\
& \rho_{f}(z)=\left(\rho_{c}-\rho_{m}\right)\left(\frac{z}{h}+\frac{1}{2}\right)^{p}+\rho_{m}
\end{aligned}
$$

$p$ denotes the non-negative constant which describes the volume fraction. Subscripts $\mathrm{b}$ and $\mathrm{t}$ represents the bottom and top of the curved nanobeam respectively. According to aforementioned subscripts when

$z=-h / 2, E=E_{b}$ and $z=h / 2, E=E_{t}$. For the sake of simplicity the bottom and the top surface of the FG curved nanobeam is assumed to be purely steel and purely alumina, respectively. Although such a power-law relation is not approximate, the above power-law relation can be easily dominated using the variation of the volume fraction of steel and alumina from the bottom to the top in experimental tasks. The variation profile of material properties across the thickness of the beam is denoted by $p$. Based on distribution function, when $p=0$, the material is homogenous.

\section{Nonlocal Theory}

Based on Eringen nonlocal theory, the stress field at point $\mathrm{X}$ does not only depend to the strain of the same point and is also depend to strain of all other points of the body. The aforementioned fact is proven by the atomic theory of lattice dynamic and experimental observation of phonon dispersion. The stress tensor $\sigma$ at point $\mathrm{X}$ is calculated as follows: 


$$
\bar{\sigma}=\int_{\Omega} K\left(\left|X^{\prime}-X, \tau\right|\right) \sigma\left(X^{\prime}\right) d X^{\prime}
$$

$\sigma$ represents the classical, microscopic second Piola-Kirchhoff stress tensor at point X, the kernel function $K\left|\left(\mathrm{X}^{\prime}-\mathrm{X}\right), \tau\right|$ denotes the nonlocal modulus, $\left(\mathrm{X}^{\prime}-\mathrm{X}\right)$ is the distance and $\tau$ stands for material parameter which depends on internal and external characteristic length. Based on the generalized Hooke's law the macroscopic stress $\sigma$ at point $X$ in Hookean solid is related to the strain at point $\mathrm{X}$ which is as follows:

$$
\sigma(X)=C(X): \varepsilon(X)
$$

The fourth-order elasticity tensor which denotes double-dot product is represented by $C$. Eqs. (2)and (3) together represent the nonlocal constitutive behavior of Hookean solid. The weighted average of the contributions of the strain

field of all points in the body to the stress field at point Xis indicated by Eq. (2). For the sake of simplicity, an

equivalent differential model is used instead of integral constitutive relation, which is evaluated as follows:

$$
\left(1-\mu \nabla^{2}\right) \bar{\sigma}=\sigma, \quad \mu=\tau^{2} \ell^{2}=e_{0}^{2} a^{2}
$$

$\tau$ is determined by $\tau=e_{0} a / \ell$ where $e_{0}$ is a constant which varies based on each material and $a_{\text {and }} \ell$ represents the internal and external characteristic length. The nonlocal parameter which is represented by ${ }^{\mu}$ varies in accordance with

different materials. For an elastic material in the one dimensional case, the nonlocal constitutive relations may be simplified as:

$$
\sigma(x)-\left(e_{0} a\right)^{2} \frac{\partial^{2} \sigma(x)}{\partial x^{2}}=E \varepsilon(x)
$$

E is the Young's modulus. For Timoshenko nonlocal FG beam, Eq. (5) can be written as:

$$
\sigma_{x x}-\mu \frac{\partial^{2} \sigma_{x x}}{\partial x^{2}}=E(z) \varepsilon_{x x}
$$

\section{Governing Equations}

Using the Hamilton principle and energy approach the governing equations are obtained in this section. For this purpose assume a curved beam with radius $R$ and angle $\alpha$ which is shown in Fig. 1. According to the Timoshenko beam theory the displacement fields for curved beam are as follows:

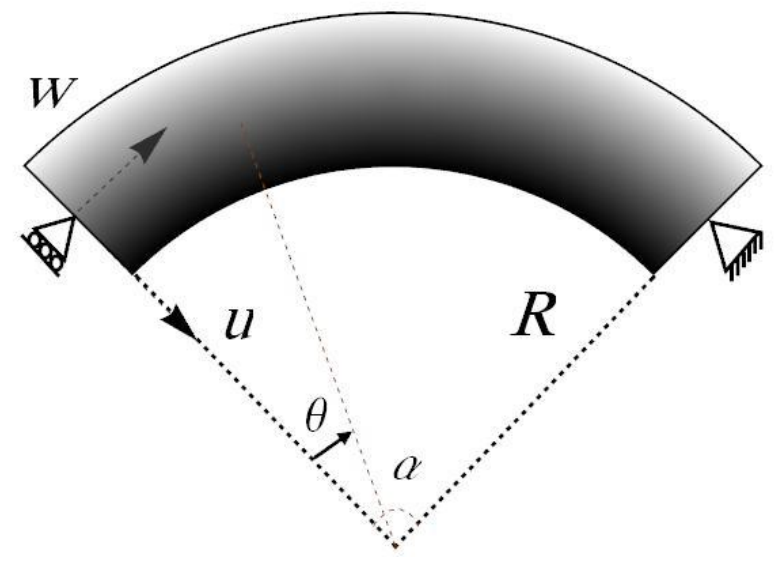

Figure 1. Schematic of functionally graded curved beam

$$
\begin{aligned}
& u_{s}(s, z, t)=-\left(1+\frac{z}{R}\right) u(s, t)-z \varphi(s, t) \\
& u_{z}(s, z, t)=-w(s, t)
\end{aligned}
$$

Where $u$ and ${ }^{w}$ denotes the radial and tangential displacements at any points of the curved beam,

respectively. ${ }^{u_{0}}$ and ${ }^{w_{0}}$ represents the displacement at the centerline and $\psi$ shows the torsionat the cross section. The strains and curves at the centerline of a curved nanobeam are calculated as follows:

$$
\varepsilon_{s s}^{0}=\frac{\partial u}{\partial s}-\frac{w}{R}
$$




$$
\gamma_{s z}=\frac{\partial u}{\partial s}-\varphi+\frac{\partial \varphi}{\partial s}
$$

Where the shear strain of bending axis is denoted by $\gamma$ and the strain at any points is evaluated as:

$$
\varepsilon_{s s}=\varepsilon_{s s}^{0}+z \kappa
$$

Hamilton principle is defined in $t=0$ and $t=T$ as follows:

$$
\int_{0}^{t}(\delta U-\delta K+\delta V) d t=0
$$

Where $U$ shows the total strain energy, $K$ denotes the Kinetic energy and $W$ is the performed work. The first variation of the total strain energy is calculated as:

$$
\begin{aligned}
& \delta U=\int_{v} \sigma_{i j} \delta \varepsilon_{i j} d V=\int_{v}\left(\sigma_{s s} \delta \varepsilon_{s s}+\sigma_{s z} \delta \gamma_{s z}\right) d V= \\
& \int_{0}^{L}\left(N\left(\frac{\delta u}{R}+\frac{\partial \delta w}{\partial s}\right)+M\left(\frac{\partial \delta \varphi}{\partial s}\right)+Q\left(\frac{\partial \delta w}{\partial s}-\delta \varphi+\frac{\delta w}{R}\right)\right) d s
\end{aligned}
$$

Where $N, M$ and $Q$ are the axial normal force, the bending moment and the shear force respectively which are defined as follows:

$$
N=\int_{A} \sigma_{s s} d A, \quad M=\int_{A} \sigma_{s s} z d A, \quad Q=\int_{A} K_{\text {shear }} \sigma_{s z} d A
$$

Where $k_{\text {shear }}$ is the shear correction coefficient. The first variation of the performed work is evaluated as follows:

$$
\delta V=-\int(f \delta u+p \delta w) d s
$$

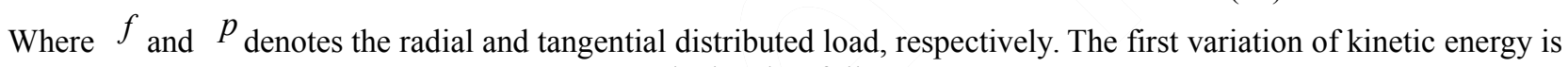
calculated as follows:

$$
\begin{aligned}
& \delta K=\frac{1}{2} \int_{0}^{L} \delta \int_{A} \rho(z)\left(\left(\frac{\partial u_{s}}{\partial t}\right)^{2}+\left(\frac{\partial u_{z}}{\partial t}\right)^{2}\right) d A d s \delta T=\int_{0}^{L}\left(I_{0}\left(\frac{\partial u}{\partial t} \frac{\partial \delta u}{\partial t}\right)+\right. \\
& \left.\left(I_{0}+\frac{2 I_{1}}{R}+\frac{I_{2}}{R^{2}}\right) \frac{\partial w}{\partial t} \frac{\partial \delta w}{\partial t}+\left(I_{1}+\frac{I_{2}}{R}\right)\left(\frac{\partial \varphi}{\partial t} \frac{\partial \delta w}{\partial t}+\frac{\partial w}{\partial t} \frac{\partial \delta \varphi}{\partial t}\right)+I_{2} \frac{\partial \varphi}{\partial t} \frac{\partial \delta \varphi}{\partial t}\right) d s
\end{aligned}
$$

Substituting Eqs. (11), (13) and (14) into Eq. (10) gives:

$$
\begin{aligned}
& \int_{0}^{T}\left(\int_{0}^{L}\left(N\left(\frac{\delta u}{R}+\frac{\partial \delta w}{\partial s}\right)+M\left(\frac{\partial \delta \varphi}{\partial s}\right)+Q\left(\frac{\partial \delta w}{\partial s}-\delta \varphi+\frac{\delta w}{R}\right)\right) d s-\right. \\
& \int_{0}^{L}\left(I_{0}\left(\frac{\partial u}{\partial t} \frac{\partial \delta u}{\partial t}\right)+\left(I_{0}+\frac{2 I_{1}}{R}+\frac{I_{2}}{R^{2}}\right) \frac{\partial w}{\partial t} \frac{\partial \delta w}{\partial t}+\left(I_{1}+\frac{I_{2}}{R}\right)\left(\frac{\partial \varphi}{\partial t} \frac{\partial \delta w}{\partial t}+\frac{\partial w}{\partial t} \frac{\partial \delta \varphi}{\partial t}\right)+I_{2} \frac{\partial \varphi}{\partial t} \frac{\partial \delta \varphi}{\partial t}\right) d s \\
& \left.-\int_{0}^{L}(f \delta u+p \delta w) d s\right) d t=0
\end{aligned}
$$

Using part by part integration, differential equation and related boundary conditions are obtained:

$$
\begin{aligned}
& \int_{0}^{T}\left(\int _ { 0 } ^ { L } \left(\left(-\frac{N}{R}+\frac{\partial Q}{\partial s}-f+I_{0} \frac{\partial^{2} u}{\partial t^{2}}\right) \delta u+\left(-\frac{\partial N}{\partial s}+\frac{Q}{R}-p+\left(I_{0}+\frac{2 I_{1}}{R}+\frac{I_{2}}{R^{2}}\right) \frac{\partial^{2} w}{\partial t^{2}}+\left(I_{1}+\frac{I_{2}}{R}\right) \frac{\partial^{2} \varphi}{\partial t^{2}}\right) \delta w+\right.\right. \\
& \left.\left.\left(-\frac{\partial M}{\partial s}-\mathrm{Q}+\left(I_{1}+\frac{I_{2}}{R}\right) \frac{\partial^{2} w}{\partial t^{2}}+I_{2} \frac{\partial^{2} \varphi}{\partial t^{2}}\right) \delta \varphi\right) d s\right) d t-\int_{0}^{T}[N \delta w+M \delta \varphi+Q \delta u]_{0}^{L} d t=0
\end{aligned}
$$

Considering Eq. (16) the differential equation of motion is calculated as follows:

$$
\frac{N}{R}+\frac{\partial Q}{\partial s}=I_{0} \frac{\partial^{2} u}{\partial t^{2}}
$$




$$
\begin{aligned}
& \frac{\partial N}{\partial s}-\frac{Q}{R}=\left(I_{0}+\frac{2 I_{1}}{R}+\frac{I_{2}}{R^{2}}\right) \frac{\partial^{2} w}{\partial t^{2}}+\left(I_{1}+\frac{I_{2}}{R}\right) \frac{\partial^{2} \varphi}{\partial t^{2}} \\
& \frac{\partial M}{\partial s}+Q=\left(I_{1}+\frac{I_{2}}{R}\right) \frac{\partial^{2} w}{\partial t^{2}}+I_{2} \frac{\partial^{2} \varphi}{\partial t^{2}}
\end{aligned}
$$

Substituting nonlocal parameter into Eq. (12), axial normal force, bending moment and shear force leads to:

$$
\begin{aligned}
& N-\mu^{2} \frac{\partial^{2} N}{\partial s^{2}}=A\left(-\frac{u}{R}+\frac{\partial w}{\partial s}\right)+B \frac{\partial \varphi}{\partial s} \\
& M-\mu^{2} \frac{\partial^{2} M}{\partial s^{2}}=B\left(-\frac{u}{R}+\frac{\partial w}{\partial s}\right)+D \frac{\partial \varphi}{\partial s} \\
& Q-\mu^{2} \frac{\partial^{2} Q}{\partial s^{2}}=K_{\text {shear }} C\left(\frac{w}{R}+\frac{\partial u}{\partial s}-\varphi\right) \\
& \left(I_{0}, I_{1}, I_{2}\right)=\int_{A} \rho(z)\left(1, z, z^{2}\right) d A(A, B, D)=\int_{A} E(z)\left(1, z, z^{2}\right) d A \text {, and } \\
& C=\int_{A} \frac{E(z)}{1+v(z)} d A
\end{aligned}
$$

Substituting Eqs.(17), (18) and (19) into nonlocal Eqs. (20), (21) and (22), the differential equation per displacement in the nonlocal theory for a curved FG nanobeam is obtained as follows:

$$
\begin{aligned}
& A\left(-\frac{u}{R^{2}}+\frac{\partial w}{R \partial s}\right)+B \frac{\partial \varphi}{R \partial s}+K_{\text {shear }} C\left(\frac{\partial w}{R \partial s}+\frac{\partial^{2} u}{\partial s^{2}}-\frac{\partial \varphi}{\partial s}\right)=I_{0} \frac{\partial^{2} u}{\partial t^{2}}-\mu^{2} I_{0} \frac{\partial^{4} u}{\partial t^{2} \partial s^{2}} \\
& A\left(-\frac{\partial u}{R \partial s}+\frac{\partial^{2} w}{\partial s^{2}}\right)+B \frac{\partial^{2} \varphi}{\partial s^{2}}-K_{\text {shear }} C\left(\frac{w}{R^{2}}+\frac{\partial u}{R \partial s}-\frac{\varphi}{R}\right)= \\
& \left(I_{0}+\frac{2 I_{1}}{R}+\frac{I_{2}}{R^{2}}\right) \frac{\partial^{2} w}{\partial t^{2}}-\mu^{2}\left(I_{0}+\frac{2 I_{1}}{R}+\frac{I_{2}}{R^{2}}\right) \frac{\partial^{4} w}{\partial s^{2} \partial t^{2}}+\left(I_{1}+\frac{I_{2}}{R}\right) \frac{\partial^{2} \varphi}{\partial t^{2}}- \\
& \mu^{2}\left(I_{1}+\frac{I_{2}}{R}\right) \frac{\partial^{4} \varphi}{\partial s^{2} \partial t^{2}} \\
& B\left(-\frac{\partial u}{R \partial s}+\frac{\partial^{2} w}{\partial s^{2}}\right)+D \frac{\partial^{2} \varphi}{\partial s^{2}}+K_{\text {shear }} C\left(\frac{w}{R}+\frac{\partial u}{\partial s}-\varphi\right)= \\
& \left(I_{1}+\frac{I_{2}}{R}\right) \frac{\partial^{2} w}{\partial t^{2}}-\mu^{2}\left(I_{1}+\frac{I_{2}}{R}\right) \frac{\partial^{4} w}{\partial s^{2} \partial t^{2}}+I_{2} \frac{\partial^{2} \varphi}{\partial t^{2}}-\mu^{2} I_{2} \frac{\partial^{4} \varphi}{\partial s^{2} \partial t^{2}}
\end{aligned}
$$

\section{Analytical Solution of Curved FG Nanobeam}

An analytical solution of a curved FG nanobeam in simply supported boundary condition is proposed in this section. The displacement was chosen in a way that satisfies the boundary condition as follows:

$$
\begin{aligned}
w(s, t) & =\sum_{n=1}^{\infty} W_{n} \cos \left(\frac{n \pi}{L} s\right) e^{i \omega_{n} t} \\
u(s, t) & =\sum_{n=1}^{\infty} U_{n} \sin \left(\frac{n \pi}{L} s\right) e^{i \omega_{n} t} \\
\varphi(s, t) & =\sum_{n=1}^{\infty} \varphi_{n} \cos \left(\frac{n \pi}{L} S\right) e^{i \omega_{n} t}
\end{aligned}
$$


Where ${ }^{i}=\sqrt{-1}, u_{n}, \mathrm{w}_{n}$ and $\varphi_{n}$ are Fourier coefficients that are obtained for different values of mode number.

\section{BendingAnalysis}

Variables $u_{0}, w_{0}$ and $\varphi$ are time independence in static analysis where $u_{0}=u_{0}(s), w_{0}=w_{0}(s)$ and $\varphi=\varphi(s)$. As a consequence all the time derivatives in Eqs. (24), (25) and (26) are eliminated. It is assumed that only the radial distributed force is applied to the curved nanobeam which is expanded based in Fourier expansion that is calculated in the following equation:

$$
f(\mathrm{~s})=\sum_{n=1}^{\infty} F_{n} \sin \left(\frac{n \pi}{L} s\right)
$$

The Fourier coefficient is calculated as follows:

$$
F_{n}=\frac{2}{L} \int_{0}^{L} f(s) \sin \left(\frac{n \pi}{L} s\right) d s
$$

For different types of loading $F_{n}$ is calculated as follows:

$$
\begin{aligned}
& f(s)=f_{0}, \quad F_{n}=\frac{4 f_{0}}{n \pi}, \quad n=1,3,5, \ldots \\
& f(s)=\frac{f_{0} s}{L}, \quad F_{n}=\frac{2 f_{0}}{n \pi}(-1)^{n+1}, \quad n=1,2,3, \ldots \\
& f(s)=f_{0} \delta\left(s-s_{0}\right) \frac{s}{L}, \quad F_{n}=2 f_{0} \sin \left(\frac{n \pi}{L} s_{0}\right), \quad n=1,2,3, \ldots \\
& f(s)=f_{0} \sin \left(\frac{\pi s}{L}\right), \quad F_{1}=f_{0}, F_{n}=0, \quad n=2,3, \ldots
\end{aligned}
$$

Substituting Eqs. (27)-(30) into Eqs. (24), (25) and (26) and eliminating the time dependent terms, the following matrix is obtained:

$$
\left(\begin{array}{lll}
k_{11} & k_{12} & k_{13} \\
k_{21} & k_{22} & k_{23} \\
k_{31} & k_{32} & k_{33}
\end{array}\right)\left[\begin{array}{l}
U_{n} \\
W_{n} \\
\varphi_{n}
\end{array}\right]=\left[\begin{array}{l}
F_{n} \lambda_{n} \\
0 \\
0
\end{array}\right]
$$

Where the stiffness matrix and $\lambda_{n}$ is calculated as follows:

$$
\begin{aligned}
& k_{11}=-\frac{A}{R^{2}}-C k_{\text {shear }}\left(\frac{n \pi}{L}\right)^{2}, \quad k_{12}=k_{21}=-\frac{A}{R}\left(\frac{n \pi}{L}\right)-\frac{C k_{\text {shear }}}{R^{2}}\left(\frac{n \pi}{L}\right), \\
& k_{13}=k_{31}=-\frac{B}{R}\left(\frac{n \pi}{L}\right)+C k_{\text {shear }}\left(\frac{n \pi}{L}\right), \quad k_{22}=-A\left(\frac{n \pi}{L}\right)^{2}-\frac{C k_{\text {shear }}}{R^{2}}, \\
& k_{23}=k_{32}=-B\left(\frac{n \pi}{L}\right)^{2}+\frac{C k_{\text {shear }}}{R}, \quad k_{33}=-D\left(\frac{n \pi}{L}\right)^{2}-C k_{\text {shear }}, \\
& \lambda_{n}=-\left(1+\mu^{2}\left(\frac{n \pi}{L}\right)^{2}\right)
\end{aligned}
$$

Solving the abovementioned equation, $u_{n}, w_{n}$ and $\varphi_{n}$ are obtained as:

$$
\begin{gathered}
U_{n}=\frac{F_{n} \lambda_{n}\left(k_{23}^{2}-k_{22} k_{33}\right)}{\xi} \\
W_{n}=\frac{F_{n} \lambda_{n}\left(-k_{13} k_{23}+k_{12} k_{33}\right)}{\xi}
\end{gathered}
$$




$$
\begin{gathered}
\psi_{n}=\frac{F_{n} \lambda_{n}\left(k_{13} k_{22}-k_{12} k_{33}\right)}{\xi} \\
\text { Where }{ }^{\xi} \text { is calculated as follows: } \\
\xi=k_{13}^{2} k_{22}-2 k_{12} k_{13} k_{23}+k_{12}^{2} k_{33}+k_{11}\left(k_{23}^{2}-k_{22} k_{33}\right)
\end{gathered}
$$

\section{Vibration Analysis}

The free vibration of a curved FG nanobeam is analyzed in this section. The radial and tangential forces are not considered in this analysis. Substituting Eqs. (27)-(29) into Eqs. (24)-(26) leads to:

$$
\begin{aligned}
& {\left[\left(\begin{array}{lll}
k_{11} & k_{12} & k_{13} \\
k_{21} & k_{22} & k_{23} \\
k_{31} & k_{32} & k_{33}
\end{array}\right)+\omega_{n}^{2}\left(\begin{array}{lll}
m_{11} & m_{12} & m_{13} \\
m_{21} & m_{22} & m_{23} \\
m_{31} & m_{32} & m_{33}
\end{array}\right]\right]\left[\begin{array}{l}
U_{n} \\
W_{n} \\
\varphi_{n}
\end{array}\right]=\left[\begin{array}{l}
0 \\
0 \\
0
\end{array}\right]} \\
& m_{11}=I_{0}+I_{0} \mu^{2}\left(\frac{n \pi}{L}\right)^{2}, \quad m_{12}=m_{21}=0, \quad m_{13}=m_{31}=0, \\
& m_{22}=I_{0}+\frac{2 I_{1}}{R}+\frac{I_{2}}{R^{2}}+\mu^{2}\left(I_{0}+\frac{2 I_{1}}{R}+\frac{I_{2}}{R^{2}}\right)\left(\frac{n \pi}{L}\right)^{2}, \\
& m_{23}=m_{32}=I_{1}+\frac{I_{2}}{R}+\mu^{2}\left(I_{1}+\frac{I_{2}}{R}\right)\left(\frac{n \pi}{L}\right)^{2}, \quad m_{33}=I_{2}+I_{2} \mu^{2}\left(\frac{n \pi}{L}\right)^{2} .
\end{aligned}
$$

In order to obtain the non-trivial solution for $U_{n} \neq 0, W_{n} \neq 0$ and $\psi_{n} \neq 0$, it is necessary to set the determinant of

$$
\left|[k]+\omega^{2}[m]\right|=0 \text { equal to zero. }
$$

\section{Results and Discussion}

Several numerical examples to study the influence of the opening angle parameter, nonlocal parameter, thickness ratio, material distribution, and mode numbers are presented in this section. The material properties of Steel and Alumina $\left(\mathrm{Al}_{2} \mathrm{O}_{3}\right.$ ) are utilized in this work. For steel $\mathrm{E}=210 \mathrm{GPa}, \rho=7800 \mathrm{Kg} / \mathrm{m}^{3}$ and for Alumina $\mathrm{E}=390 \mathrm{GPa}, \rho=3960 \mathrm{Kg} / \mathrm{m}^{3}$.

\begin{tabular}{|c|c|c|c|c|c|c|c|c|c|}
\hline \multirow[t]{3}{*}{$\mathbf{L} / \mathbf{h}$} & \multirow[t]{3}{*}{$\mu$} & \multicolumn{2}{|l|}{$\mathbf{P}$} & & & & & & \\
\hline & & \multicolumn{2}{|l|}{ o } & \multicolumn{2}{|l|}{0.5} & \multicolumn{2}{|l|}{1} & \multicolumn{2}{|l|}{10} \\
\hline & & Present & $\begin{array}{l}\text { Rahmani } \\
\text { et al }\end{array}$ & Present & $\begin{array}{l}\text { Rahmani } \\
\text { et a } \text { ll }^{24]}\end{array}$ & Present & $\begin{array}{l}\text { Rahmani } \\
\text { et a al }{ }^{24]}\end{array}$ & Present & $\begin{array}{l}\text { Rahmani } \\
\text { et al } l^{[24]}\end{array}$ \\
\hline \multirow[t]{5}{*}{20} & 0 & 9.82945 & 9.8296 & 7.71442 & 7.7149 & 6.96707 & 6.9676 & 5.65187 & 5.6521 \\
\hline & $1 \times 10^{-12}$ & 9.37758 & 9.3777 & 7.35978 & 7.3602 & 6.64678 & 6.6473 & 5.39204 & 5.3923 \\
\hline & $2 \times 10^{-12}$ & 8.98279 & 8.9829 & 7.04994 & 7.0504 & 6.36695 & 6.3674 & 5.16504 & 5.1653 \\
\hline & $3 \times 10^{-12}$ & 8.63400 & 8.6341 & 6.77620 & 6.7766 & 6.11973 & 6.1202 & 4.96449 & 4.9647 \\
\hline & $4 \times 10^{-12}$ & 8.32292 & 8.3230 & 6.53206 & 6.5325 & 5.89924 & 5.8997 & 4.78562 & 4.7858 \\
\hline
\end{tabular}

9.Validation

As mentioned, when the radius of curved FG nanobeam and its opening angle increases and decreases, its mechanical behavior approaches to those of straight one. For this purpose a comparison has been made between the

vibration behavior of a curved FG nanobeam with very slight opening angle of $\alpha=\frac{\pi}{360}=0.5$ and a straight nanobeam $^{[24]}$.The dimensionless natural frequency $\left(\bar{\omega}=\omega \times L^{2} \sqrt{\rho_{c} A / E_{c} I}\right)$ of a curved FG nanobeam with very slight opening angle and a straight nanobeam for $L=10000 \times 10^{-9}, b=10000 \times 10^{-9}$ and $k_{s}=\frac{5}{6}$ is compared in Table 1.It is concluded that our results are in agood agreement with Ref. ${ }^{[2]]}$. 


\begin{tabular}{|l|l|l|l|l|l|l|l|l|l|}
\hline & $5 \times 10^{-12}$ & 8.04321 & 8.0433 & 6.31253 & 6.3129 & 5.70099 & 5.7014 & 4.62479 & 4.6250 \\
\hline 50 & 0 & 9.86304 & 9.8631 & 7.74106 & 7.7413 & 6.99147 & 6.9917 & 5.67283 & 5.6730 \\
\hline & $1 \times 10^{-12}$ & 9.40962 & 9.4097 & 7.38519 & 7.3854 & 6.67006 & 6.6703 & 5.41204 & 5.4122 \\
\hline & $2 \times 10^{-12}$ & 9.01349 & 9.0136 & 7.07428 & 7.0745 & 6.38926 & 6.3895 & 5.18420 & 5.1843 \\
\hline & $3 \times 10^{-12}$ & 8.66351 & 8.6636 & 6.79960 & 6.7998 & 6.14117 & 6.1414 & 4.98290 & 4.9830 \\
\hline & $4 \times 10^{-12}$ & 8.35136 & 8.3515 & 6.55461 & 6.5548 & 5.91991 & 5.9201 & 4.80337 & 4.8035 \\
\hline & $5 \times 10^{-12}$ & 8.07070 & 8.0708 & 6.33433 & 6.3345 & 5.72096 & 5.7212 & 4.64195 & 4.6421 \\
\hline 100 & 0 & 9.86788 & 9.8680 & 7.74494 & 7.7451 & 6.99505 & 6.9952 & 5.67587 & 5.6760 \\
\hline & $1 \times 10^{-12}$ & 9.41425 & 9.4143 & 7.38891 & 7.3891 & 6.67347 & 6.6736 & 5.41494 & 5.4150 \\
\hline & $2 \times 10^{-12}$ & 9.01792 & 9.0180 & 7.07784 & 7.0780 & 6.39252 & 6.3927 & 5.18698 & 5.1871 \\
\hline & $3 \times 10^{-12}$ & 8.66775 & 8.6678 & 6.80301 & 6.8032 & 6.14430 & 6.1444 & 4.98557 & 4.9857 \\
\hline & $4 \times 10^{-12}$ & 8.35547 & 8.3555 & 6.55791 & 6.5580 & 5.92293 & 5.9231 & 4.80594 & 4.8060 \\
\hline & $5 \times 10^{-12}$ & 8.07466 & 8.0747 & 6.33752 & 6.3376 & 5.72388 & 5.7240 & 4.64444 & 4.6445 \\
\hline
\end{tabular}

Table 1. Dimensionless frequencies for firs mode

\section{Free Vibration of Curved FG Nanobeam}

The vibration of a curved FG nanobeam per different aspect ratios of 10, 25 and 75, power law indexes of $0,0.1,1$ and 10, and opening angles of $\frac{3 \pi}{4}, \frac{2 \pi}{3}, \frac{\pi}{2}, \frac{\pi}{3}, \frac{\pi}{6}, \frac{\pi}{9}$ are analyzed and non-dimensionalized in this section. The non-dimensionalized natural frequencies for nonlocal parameter $\mu=0$ are represented in Table 1 .

As it is clear, increasing both the power index law and the opening angle results in decreasing the amount of frequency. Increasing the thickness is also results in decreasing the frequency of curved FG nanobeam. The amount of frequency of a curved FG nanobeam for nonlocal parameters of1, 2, 3 and 4 is represented in Tables 2, 3, 4 and 5 respectively. Comparing the aforementioned tables it is clear that as the nonlocal parameter increases, the amount of non-dimensionalized frequency increases.

\begin{tabular}{|c|c|c|c|c|c|c|c|c|c|c|c|c|}
\hline \multirow[t]{2}{*}{$\alpha$} & \multicolumn{4}{|c|}{$\mathrm{L} / \mathrm{h}=10$} & \multicolumn{4}{|c|}{$L / h=25$} & \multicolumn{4}{|c|}{$L / h=75$} \\
\hline & $P=0$ & $2^{P=0 .}$ & $P=1$ & $P=5$ & $\mathbf{P}=\mathbf{0}$ & $2^{P=0 .}$ & $P=1$ & $P=5$ & $P=0$ & $2^{P=0 .}$ & $P=1$ & $P=5$ \\
\hline \multirow{2}{*}{$\pi / 9$} & 9.534 & 8.381 & 6.723 & 5.711 & 9.662 & 8.505 & 6.835 & 5.807 & 9.685 & 8.530 & 6.860 & 5.828 \\
\hline & 86 & 26 & 27 & 59 & 99 & 22 & 33 & 95 & 33 & 18 & 90 & 80 \\
\hline \multirow{2}{*}{$\pi / 6$} & 9.315 & 8.180 & 6.553 & 5.569 & 9.440 & 8.305 & 6.671 & 5.669 & 9.462 & 8.332 & 6.700 & 5.693 \\
\hline & 67 & 15 & 50 & 78 & 42 & 76 & 48 & 80 & 16 & 43 & 64 & 03 \\
\hline \multirow{2}{*}{$\pi / 3$} & 8.193 & 7.178 & 5.733 & 4.876 & 8.301 & 7.296 & 5.853 & 4.976 & 8.320 & 7.324 & 5.887 & 5.002 \\
\hline & 67 & 71 & 71 & 71 & 63 & 89 & 62 & 58 & 42 & 65 & 66 & 97 \\
\hline \multirow{2}{*}{$\pi / 2$} & 6.519 & 5.707 & 4.552 & 3.870 & 6.604 & 5.803 & 4.652 & 3.955 & 6.618 & 5.826 & 4.682 & 3.978 \\
\hline & 51 & 94 & 00 & 98 & 18 & 01 & 13 & 10 & 89 & 09 & 04 & 54 \\
\hline \multirow{2}{*}{$2 \pi / 3$} & 4.491 & 3.936 & 3.138 & 2.666 & 4.550 & 3.999 & 3.206 & 2.725 & 4.560 & 4.015 & 3.226 & 2.741 \\
\hline & 93 & 02 & 79 & 54 & 75 & 93 & 51 & 08 & 94 & 05 & 58 & 45 \\
\hline \multirow{2}{*}{$3 \pi / 4$} & 3.400 & 2.981 & 2.379 & 2.019 & 3.445 & 3.029 & 2.429 & 2.063 & 3.453 & 3.040 & 2.443 & 2.075 \\
\hline & 11 & 97 & 07 & 62 & 51 & 54 & 04 & 74 & 37 & 41 & 47 & 88 \\
\hline
\end{tabular}

Table 2. The variation of dimensionless frequencies $\bar{\omega}=\omega \times L^{2} \sqrt{\left(\rho_{c} A\right) /\left(E_{c} I\right)}$ versus opening angle, power index law and aspect ratio for simply supported curved beam $(\mu=0)$ 


\begin{tabular}{|c|c|c|c|c|c|c|c|c|c|c|c|c|}
\hline \multirow[t]{2}{*}{$\alpha$} & \multicolumn{4}{|c|}{$\mathrm{L} / \mathrm{h}=\mathbf{1 0}$} & \multicolumn{4}{|c|}{$L / h=25$} & \multicolumn{4}{|c|}{$L / h=75$} \\
\hline & $P=0$ & $2^{P=0 .}$ & $P=1$ & $P=5$ & $P=0$ & $2^{P=0 .}$ & $P=1$ & $P=5$ & $P=0$ & $2^{P=0 .}$ & $P=1$ & $P=5$ \\
\hline \multirow{2}{*}{$\pi / 9$} & 9.096 & 7.995 & 6.414 & 5.449 & 9.218 & 8.114 & 6.521 & 5.540 & 9.240 & 8.138 & 6.545 & 5.560 \\
\hline & 52 & 96 & 18 & 02 & 77 & 22 & 10 & 95 & 08 & 04 & 49 & 84 \\
\hline \multirow{2}{*}{$\pi / 6$} & 8.887 & 7.804 & 6.252 & 5.313 & 9.006 & 7.923 & 6.364 & 5.409 & 9.027 & 7.949 & 6.392 & 5.431 \\
\hline & 41 & 10 & 22 & 73 & 42 & 93 & 78 & 15 & 17 & 38 & 60 & 31 \\
\hline \multirow{2}{*}{$\pi / 3$} & 7.816 & 6.848 & 5.470 & 4.652 & 7.919 & 6.961 & 5.584 & 4.747 & 7.937 & 6.987 & 5.617 & 4.772 \\
\hline & 99 & 69 & 12 & 52 & 99 & 44 & 52 & 79 & 91 & 92 & 00 & 97 \\
\hline \multirow{2}{*}{$\pi / 2$} & 6.219 & 5.445 & 4.342 & 3.693 & 6.300 & 5.536 & 4.438 & 3.773 & 6.314 & 5.558 & 4.466 & 3.795 \\
\hline & 80 & 53 & 73 & 02 & 57 & 23 & 27 & 27 & 60 & 26 & 80 & 64 \\
\hline \multirow{2}{*}{$2 \pi / 3$} & 4.285 & 3.755 & 2.994 & 2.543 & 4.341 & 3.816 & 3.059 & 2.599 & 4.351 & 3.830 & 3.078 & 2.615 \\
\hline & 43 & 07 & 49 & 95 & 55 & 04 & 1 & 81 & 27 & 47 & 24 & 42 \\
\hline \multirow{2}{*}{$3 \pi / 4$} & 3.243 & 2.844 & 2.269 & 1.926 & 3.287 & 2.890 & 2.317 & 1.968 & 3.294 & 2.900 & 2.331 & 1.980 \\
\hline & 8 & 89 & 7 & 77 & 12 & 27 & 37 & 86 & 62 & 63 & 14 & 46 \\
\hline
\end{tabular}

Table 3. The variation of dimensionless frequencies $\bar{\omega}=\omega \times L^{2} \sqrt{\left(\rho_{c} A\right) /\left(E_{c} I\right)}$ versus opening angle, power index law and aspect ratio for simply supported curved beam $\left(\mu=1 \mathrm{~nm}^{2}\right)$

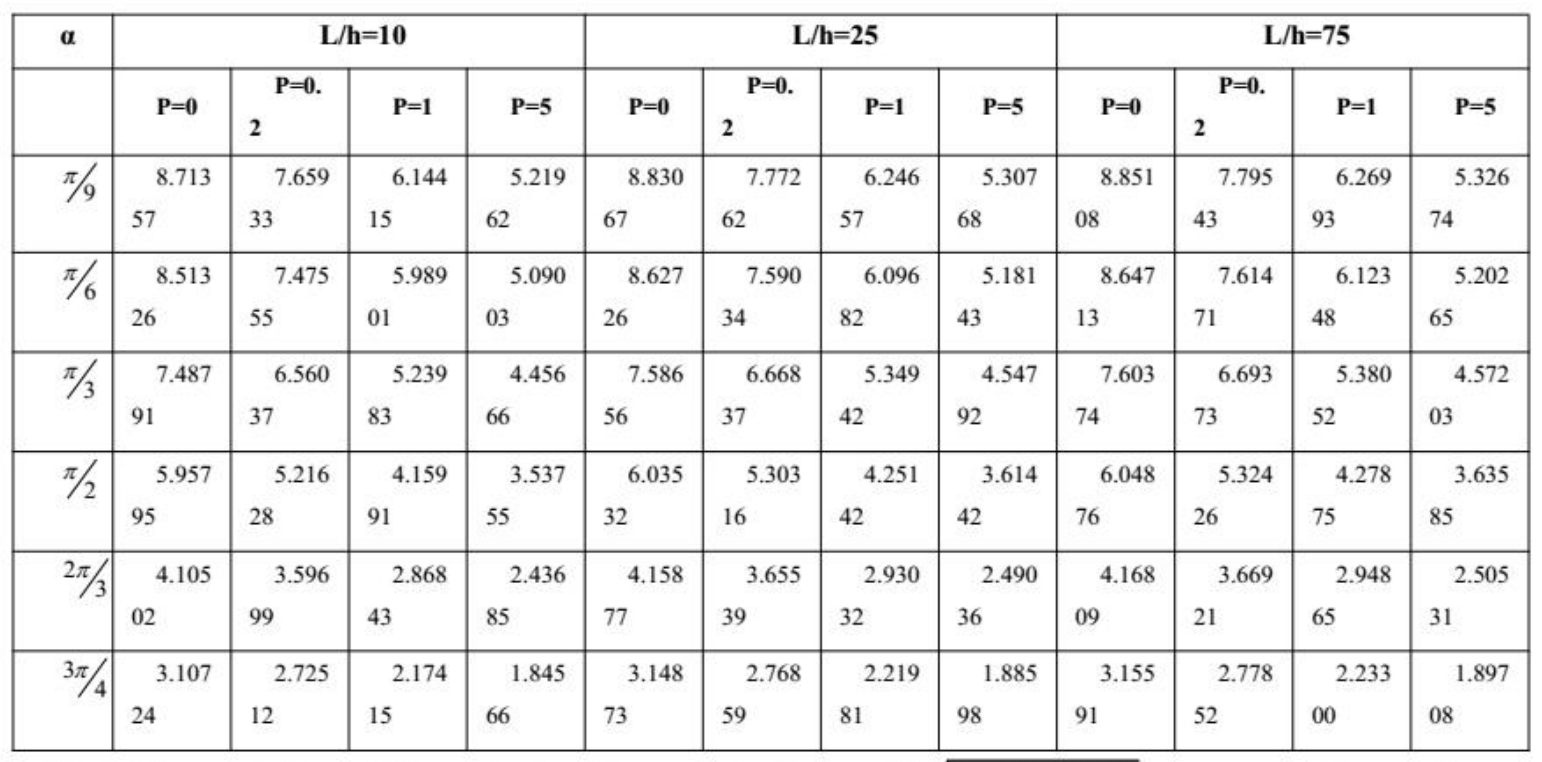

Table 4. The variation of dimensionless frequencies $\bar{\omega}=\omega \times L^{2} \sqrt{\left(\rho_{c} A\right) /\left(E_{c} I\right)}$ versus opening angle, power index law and aspect ratio for simply supported curved beam $\left(\mu=2 \mathrm{~nm}^{2}\right)$ 


\begin{tabular}{|c|c|c|c|c|c|c|c|c|c|c|c|c|}
\hline \multirow[t]{2}{*}{$\boldsymbol{\alpha}$} & \multicolumn{4}{|c|}{$L / h=10$} & \multicolumn{4}{|c|}{$L / h=25$} & \multicolumn{4}{|c|}{$L / h=75$} \\
\hline & $\mathrm{P}=0$ & $2^{P-0 .}$ & $P=1$ & $P=5$ & $P=0$ & $2^{\mathrm{P}-0 .}$ & $P=1$ & $P=5$ & $P=0$ & $2^{\mathrm{P}-0 .}$ & $P=1$ & $P-5$ \\
\hline$\pi / 9$ & $23^{8.375}$ & 93 & $\begin{array}{l}5.905 \\
59\end{array}$ & 95 & $\begin{array}{l}8.487 \\
79\end{array}$ & $\begin{array}{l}7.470 \\
82\end{array}$ & 02 & 59 & $41^{8.507}$ & $75^{7.492}$ & $\begin{array}{l}6.026 \\
48\end{array}$ & $\begin{array}{l}5.119 \\
91\end{array}$ \\
\hline$\pi / 6$ & $\begin{array}{l}8.182 \\
70\end{array}$ & $\begin{array}{l}7.185 \\
29\end{array}$ & $\begin{array}{l}5.756 \\
47^{-1}\end{array}$ & $3^{4.892}$ & $28^{8.292}$ & $62^{7.295}$ & $\begin{array}{l}5.860 \\
09\end{array}$ & 24 & $\begin{array}{l}8.311 \\
38\end{array}$ & $05^{7.319}$ & $\begin{array}{r}5.885 \\
71\end{array}$ & $\begin{array}{l}5.000 \\
64\end{array}$ \\
\hline$\pi / 3$ & $\begin{array}{l}7.197 \\
16\end{array}$ & $\begin{array}{r}6.305 \\
64\end{array}$ & $\begin{array}{l}5.036 \\
38\end{array}$ & $\begin{array}{l}4.283 \\
61\end{array}$ & 99 & $\begin{array}{l}6.409 \\
45\end{array}$ & $\begin{array}{l}5.141 \\
71\end{array}$ & $33^{4.371}$ & so & $\begin{array}{l}6.433 \\
83\end{array}$ & $\begin{array}{l}5.171 \\
61\end{array}$ & $\begin{array}{l}\quad 4.394 \\
51\end{array}$ \\
\hline$\pi / 2$ & $\begin{array}{l}5.726 \\
61\end{array}$ & $\begin{array}{r}5.013 \\
74\end{array}$ & $\begin{array}{l}3.998 \\
39\end{array}$ & $1^{3.400}$ & $\begin{array}{l}5.800 \\
98\end{array}$ & 25 & $34^{4.086}$ & $\begin{array}{l}3.474 \\
08\end{array}$ & $90^{5.813}$ & 53 & $61^{4.112}$ & $\begin{array}{l}3.494 \\
67\end{array}$ \\
\hline $2 \pi / 3$ & $\begin{array}{l}3.945 \\
63\end{array}$ & $\begin{array}{l}3.457 \\
32^{2}\end{array}$ & $05^{2.757}$ & $24^{2.342}$ & 29 & $4^{3.513}$ & $54^{2.816}$ & $\begin{array}{r}2.393 \\
66\end{array}$ & $25^{4.006}$ & $75^{3.526}$ & $16^{2.834}$ & $\begin{array}{l}2.408 \\
04\end{array}$ \\
\hline $3 \pi / 4$ & 59 & $31 \quad 2.619$ & $7^{2.089}$ & $9^{1.773}$ & $\begin{array}{l}3.026 \\
47^{3}\end{array}$ & $09^{2.661}$ & $62^{2.133}$ & $75^{1.812}$ & $\begin{array}{r}3.033 \\
38\end{array}$ & $63^{2.670}$ & $30^{2.146}$ & $42^{1.823}$ \\
\hline
\end{tabular}

Table 5. The variation of dimensionless frequencies $\bar{\omega}=\omega \times L^{2} \sqrt{\left(\rho_{c} A\right) /\left(E_{c} I\right)}$ versus opening angle, power index law and aspect ratio for simply supported curved beam $\left(\mu=3 \mathrm{~nm}^{2}\right)$

\begin{tabular}{|c|c|c|c|c|c|c|c|c|c|c|c|c|}
\hline \multirow[t]{2}{*}{$\alpha$} & \multicolumn{4}{|c|}{$\mathrm{L} / \mathrm{h}=\mathbf{1 0}$} & \multicolumn{4}{|c|}{$L / h=25$} & \multicolumn{4}{|c|}{$L / h=75$} \\
\hline & $P=0$ & $2^{P=0 .}$ & $P=1$ & $P=5$ & $P=0$ & $2^{P=0 .}$ & $P=1$ & $P=5$ & $P=0$ & $2^{P=0 .}$ & $P=1$ & $P=5$ \\
\hline \multirow{2}{*}{$\pi / 9$} & 8.073 & 7.096 & 5.692 & 4.836 & 8.181 & 7.201 & 5.787 & 4.917 & 8.200 & 7.222 & 5.809 & 4.935 \\
\hline & 48 & 69 & 81 & 19 & 97 & 65 & 70 & 78 & 89 & 79 & 35 & 44 \\
\hline \multirow{2}{*}{$\pi / 6$} & 7.887 & 6.926 & 5.549 & 4.716 & 7.993 & 7.032 & 5.648 & 4.800 & 8.011 & 7.055 & 5.673 & 4.820 \\
\hline & 88 & 41 & 07 & 12 & 51 & 76 & 96 & 81 & 92 & 35 & 65 & 47 \\
\hline \multirow[t]{2}{*}{$\pi / 3$} & 6.937 & 6.078 & 4.854 & 4.129 & 7.029 & 6.178 & 4.956 & 4.213 & 7.045 & 6.202 & 4.985 & 4.236 \\
\hline & 85 & 45 & 92 & 27 & 26 & 52 & 45 & 83 & 18 & 02 & 28 & 18 \\
\hline \multirow{2}{*}{$\pi / 2$} & 5.520 & 4.833 & 3.854 & 3.277 & 5.591 & 4.913 & 3.939 & 3.348 & 5.604 & 4.933 & 3.964 & 3.368 \\
\hline & 28 & 10 & 33 & 69 & 98 & 60 & 11 & 91 & 43 & 14 & 44 & 77 \\
\hline \multirow{2}{*}{$2 \pi / 3$} & 3.803 & 3.332 & 2.657 & 2.257 & 3.853 & 3.386 & 2.715 & 2.307 & 3.861 & 3.399 & 2.732 & 2.321 \\
\hline & 47 & 76 & 71 & 85 & 27 & 87 & 06 & 42 & 90 & 68 & 05 & 28 \\
\hline \multirow{2}{*}{$3 \pi / 4$} & 2.878 & 2.524 & 2.014 & 1.710 & 2.917 & 2.565 & 2.056 & 1.747 & 2.924 & 2.574 & 2.068 & 1.757 \\
\hline & 98 & 93 & 44 & 08 & 43 & 21 & 75 & 43 & 09 & 41 & 97 & 72 \\
\hline
\end{tabular}

Table 6. The variation of dimensionless frequencies $\bar{\omega}=\omega \times L^{2} \sqrt{\left(\rho_{c} A\right) /\left(E_{c} I\right)}$ versus opening angle, power index law and aspect ratio for simply supported curved beam $\left(\mu=4 \mathrm{~nm}^{2}\right)$

The non-dimensionalized frequency variation of a curved FG nanobeam versus the power index law for different $L / h$ values, nonlocal parameter of $\mu=1$ and different opening angles of $\frac{3 \pi}{4}, \frac{2 \pi}{3}, \frac{\pi}{2}, \frac{\pi}{3}, \frac{\pi}{6}, \frac{\pi}{9}$ is shown in Fig. 2a-2f. As seen, increasing the power index law decrease the amount of frequency which is more intense for $\mathrm{p}<2$. Also as $1 / \mathrm{h}$ ratio increases the difference between the frequencies decreases. This leads to a fact that the shear effect decreases with increasing the length of nanotube. As observed in Tables 1-5 it is also concluded that as the opening angle increases the amount of frequency decreases. 

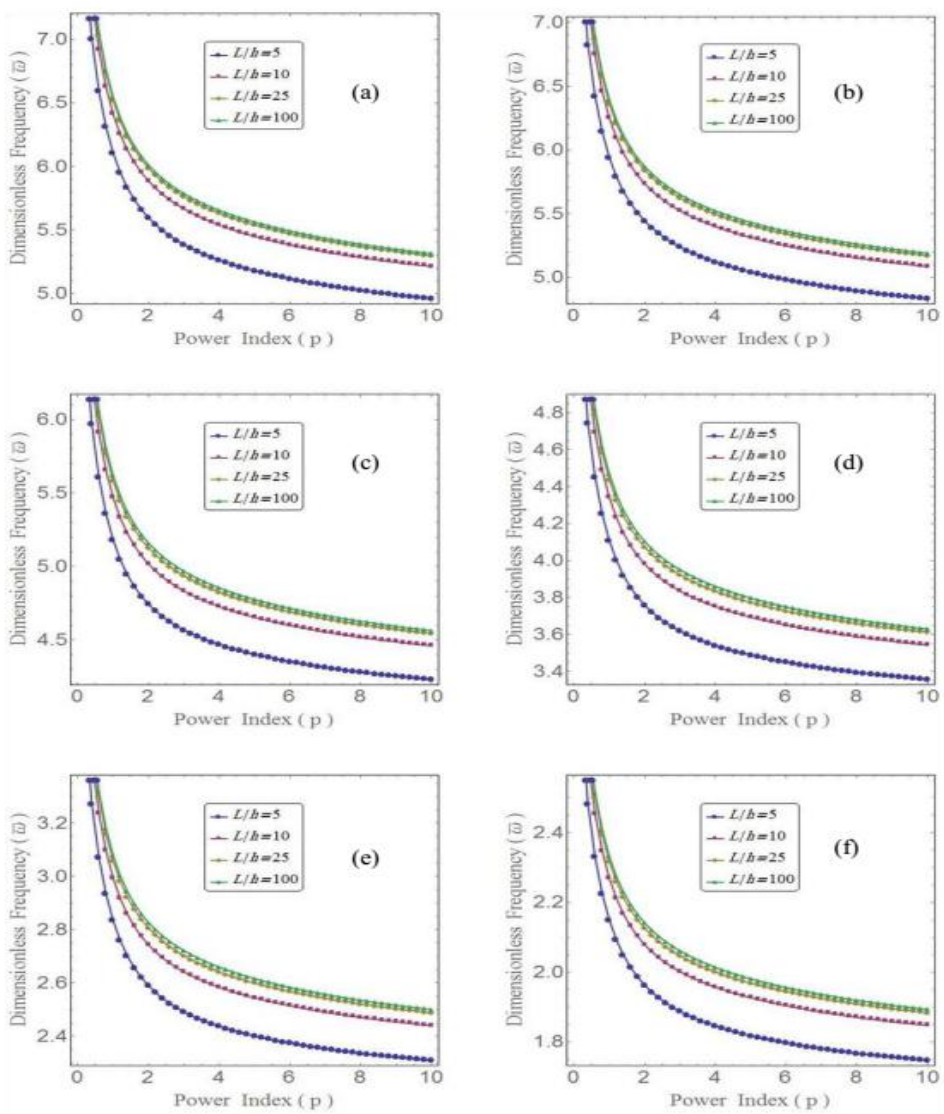

Figure 2. The variation of non-dimensionalizedfrequencies $\bar{\omega}=\omega \times L^{2} \sqrt{\left(\rho_{c} A\right) /\left(E_{c} I\right)}$ versus power law index for opening angles $\frac{\pi}{18}, \frac{\pi}{4}, \frac{\pi}{2}$ and $\frac{2 \pi}{3}$ and nonlocal parameters of $0(\mathrm{a}), 1(\mathrm{~b}), 2(\mathrm{c})$ and $3(\mathrm{~d})\left(\mathrm{nm}^{2}\right)$.

The variation of non-dimensionalized frequency per different aspect ratios versus mode number and for nonlocal parameter of $\mu=0,1,2,3$ is shown in Fig.3a-3d respectively. The opening angle is assumed to be $\frac{\pi}{3}$. As predicted increasing the mode number tends to increase the frequency values.The importance of nonlocal parameter and non-classical theories is clarified by comparing Fig.3a with Fig.3b as the frequency of the sixth mode of Fig.3a is twice those of Fig.3b.
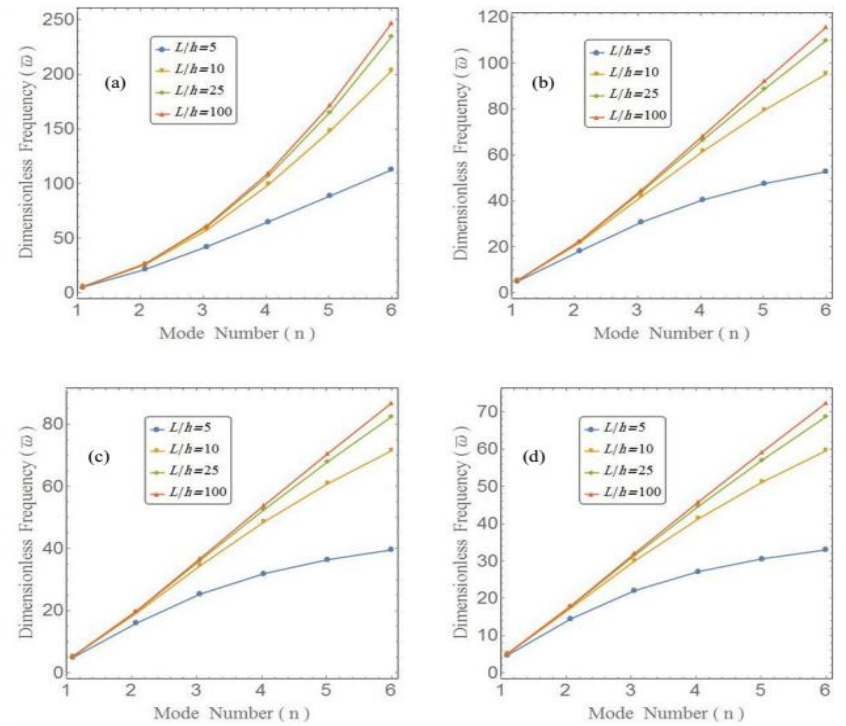

Figure 3. The variation of non-dimensionalizedfrequencies $\bar{\omega}=\omega \times L^{2} \sqrt{\left(\rho_{c} A\right) /\left(E_{c} I\right)}$ versus first six vibration modes for 
different nonlocal parameters of $0,1,2,3$ and $4\left(\mathrm{~nm}^{2}\right)$, the opening angles of $\frac{\pi}{9}$ (a), $\frac{\pi}{2}$ (b), $\frac{2 \pi}{3}$ (c) and $\frac{3 \pi}{4}$ (d).(p=1)

\section{Bending Analysisof Curved FG Nanobeam}

Bending behavior of a curved FG nanobeam, which is under the distributed radial load, is analyzed in this section. The \[ \bar{u}_{n}=\frac{100 E_{c} I}{f_{0} R^{4} \alpha^{4}} u_{n}\left(\frac{\alpha}{2}\right), \bar{W}_{n}=\frac{100 E_{c} I}{f_{0} R^{4} \alpha^{4}} w_{n}(0) \text { where } \bar{u}_{n}, \bar{w}_{n} \text { denote } \]
non-dimensionalized displacements are calculated as
the non-dimensionalized radial and tangential displacement, respectively. The non-dimensionalized displacement for the non-dimensionalized radial and tangential displacement, respectively. The non-dimensionalized displacement for
nonlocal parameters of $0,1,2,3$ and 4 is represented in tables (7)-(11). As it can be seen the amount of displacement increases as the opening angle increases which is more intense when the opening angle increases from $\frac{2 \pi}{3}$ to $\frac{3 \pi}{4}$.It is clear that increasing both the nonlocal parameter and the aspect ratio results in increasing and decreasing the non-dimensionalized radial displacement, respectively.

\begin{tabular}{|c|c|c|c|c|c|c|c|c|c|c|c|c|c|}
\hline & \multirow[t]{2}{*}{$\alpha$} & \multicolumn{4}{|c|}{$\mathrm{L} / \mathrm{h}=\mathbf{1 0}$} & \multicolumn{4}{|c|}{$\mathrm{L} / \mathrm{h}=\mathbf{2 5}$} & \multicolumn{4}{|c|}{$L / h=75$} \\
\hline & & $P=0$ & $2^{P=0 .}$ & $P=1$ & $P=5$ & $P=0$ & $2^{P=0 .}$ & $P=1$ & $P=5$ & $P=0$ & $2^{\mathrm{P}=0 .}$ & $P=1$ & $P=5$ \\
\hline \multirow{6}{*}{ 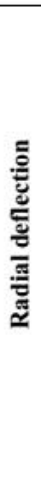 } & $\pi / 9$ & $9^{1.3668}$ & $2^{1.5173}$ & $6^{1.8375}$ & $4^{2.0962}$ & $6^{1.3400}$ & $6^{1.4869}$ & $6^{1.7983}$ & $2^{2.0473}$ & $2^{1.3355}$ & $9^{1.4814}$ & $9^{1.7906}$ & $9^{2.0379}$ \\
\hline & $\pi / 6$ & $7^{1.4109}$ & $7^{1.5672}$ & $\begin{array}{l}1.9000 \\
4^{2}\end{array}$ & $5^{2.1671}$ & $2^{1.3831}$ & $3^{1.5351}$ & $\begin{array}{ll} & 1.8574 \\
3 & \end{array}$ & $1^{2.1144}$ & 1.3784 & $9^{1.5291}$ & $2^{1.8486}$ & $6^{2.1038}$ \\
\hline & $\pi / 3$ & $3^{1.6901}$ & $4^{1.8809}$ & $2^{2.2875}$ & $3^{2.6078}$ & $1^{1.6557}$ & $3^{1.8391}$ & $3^{2.2281}$ & $7^{2.5358}$ & $8^{1.6498}$ & $6^{1.8308}$ & $5^{2.2142}$ & 2.5198 \\
\hline & $\pi / 2$ & 2.379 & $2^{2.6526}$ & $1^{3.2361}$ & $\begin{array}{l}3.6876 \\
4^{3}\end{array}$ & $1^{2.3281}$ & $6^{2.5880}$ & 3.1395 & $9^{3.5723}$ & $9^{2.3194}$ & 2.5746 & $9^{3.1150}$ & $8^{3.5446}$ \\
\hline & $2 \pi / 3$ & $\begin{array}{l}4.3474 \\
5\end{array}$ & $6^{4.8565}$ & $6^{5.9432}$ & 6.7699 & $2^{4.2483}$ & $6^{4.7263}$ & 5.7408 & $9^{6.5310}$ & $4^{4.2315}$ & $\begin{array}{l}4.6981 \\
7^{1}\end{array}$ & $\begin{array}{ll} & 5.6869 \\
3 & \end{array}$ & $4^{6.4707}$ \\
\hline & $3 \pi / 4$ & $8^{7.0207}$ & $\begin{array}{ll} & 7.8502 \\
5 & \end{array}$ & $3^{9.6216}$ & $9^{10.957}$ & $2^{6.8548}$ & $\begin{array}{l}7.6291 \\
3\end{array}$ & $6^{9.2725}$ & 10.548 & $3^{6.8267}$ & $\begin{array}{l}7.5805 \\
5\end{array}$ & $9^{9.1778}$ & $5^{10.442}$ \\
\hline \multirow{6}{*}{ 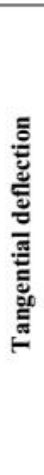 } & $\pi / 9$ & $377^{0.2542}$ & $32^{0.2723}$ & $311^{0.3098}$ & $388^{0.3542}$ & $\begin{array}{l}0.2500 \\
89\end{array}$ & $36^{0.2732}$ & $53^{0.3214}$ & $\begin{array}{l}0.3647 \\
07\end{array}$ & $\begin{array}{l}0.2493 \\
86\end{array}$ & $\begin{array}{l}0.2748 \\
87\end{array}$ & $02^{0.3282}$ & $38^{0.3713}$ \\
\hline & $\pi / 6$ & $4^{0.3935}$ & $\begin{array}{l}0.4260 \\
97\end{array}$ & $\begin{array}{l}0.4940 \\
99\end{array}$ & $\begin{array}{l}0.5630 \\
72\end{array}$ & $\begin{array}{l}0.3871 \\
21\end{array}$ & $\begin{array}{l}0.4247 \\
7^{0}\end{array}$ & $\begin{array}{l}0.5033 \\
91\end{array}$ & $\begin{array}{l}0.5704 \\
38\end{array}$ & $\begin{array}{l}0.3860 \\
34\end{array}$ & $14^{0.4261}$ & $\begin{array}{l}0.5099 \\
7^{2}\end{array}$ & $72^{0.5767}$ \\
\hline & $\pi / 3$ & $4^{0.9413}$ & 1.0297 & $2^{1.2153}$ & $3^{1.3808}$ & $22^{0.9260}$ & $\begin{array}{l}1.0202 \\
7^{2}\end{array}$ & $2^{1.2175}$ & $2^{1.3781}$ & $\begin{array}{l}0.9234 \\
27\end{array}$ & 1.0207 & $5^{1.2243}$ & $2^{1.3842}$ \\
\hline & $\pi / 2$ & $9^{1.9826}$ & $1^{2.1753}$ & $2^{2.5805}$ & $5^{2.9294}$ & 1.9505 & $3^{2.1516}$ & $1^{2.5728}$ & $1^{2.9112}$ & $5^{1.9450}$ & $1^{2.1508}$ & $7^{2.5816}$ & $5^{2.9184}$ \\
\hline & $2 \pi / 3$ & $\begin{array}{l}4.8158 \\
7^{4}\end{array}$ & $1^{5.2901}$ & $8^{6.2882}$ & $1^{7.1361}$ & $\begin{array}{l}4.7379 \\
3\end{array}$ & $4^{5.2290}$ & $1^{6.2577}$ & $\begin{array}{ll} & 7.0798 \\
3 & \end{array}$ & $3^{4.7247}$ & $9^{5.2253}$ & $6^{6.2738}$ & $8^{7.0919}$ \\
\hline & $3 \pi / 4$ & $4^{8.7344}$ & $7^{9.5974}$ & $2^{11.414}$ & 12.952 & 8.5933 & $2^{9.4852}$ & $5^{11.353}$ & $6^{12.844}$ & $1^{8.5694}$ & $6^{9.4778}$ & $4^{11.380}$ & $2^{12.864}$ \\
\hline
\end{tabular}

Table 7. The variation of dimensionless radial and tangential displacement versus opening angle, aspect ratios and power law index for simply supported curved beam ( $\mu=0$ 


\begin{tabular}{|c|c|c|c|c|c|c|c|c|c|c|c|c|c|}
\hline & $\alpha$ & & & $1=10$ & & & & $\mathrm{a}=25$ & & & & $h=75$ & \\
\hline & & $\mathrm{P}=\mathbf{0}$ & $2^{P=0 .}$ & $P=1$ & $\mathrm{P}=5$ & $\mathrm{P}=\mathbf{0}$ & $2^{P=0 .}$ & $P=1$ & $\mathrm{P}=5$ & $\mathrm{P}=0$ & $2^{\mathrm{P}=0 .}$ & $P=1$ & $P=5$ \\
\hline \multirow{6}{*}{ 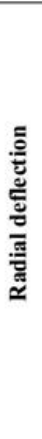 } & $\pi / 9$ & $8^{1.4976}$ & $2^{1.6625}$ & $9^{2.0133}$ & $4^{2.2967}$ & 1.4687 & 1.6297 & 1.971 & $4^{2.2438}$ & 1.4638 & $9^{1.6237}$ & $9^{1.9626}$ & $4^{2.2337}$ \\
\hline & $\pi / 6$ & $9^{1.5460}$ & $7^{1.7173}$ & $1^{2.0820}$ & $1^{2.3746}$ & $9^{1.5159}$ & $2^{1.6826}$ & $7^{2.0358}$ & $3^{2.3175}$ & 1.5109 & $8^{1.6761}$ & $1^{2.0263}$ & $9^{2.3060}$ \\
\hline & $\pi / 3$ & $1^{1.8527}$ & $9^{2.0618}$ & $9^{2.5075}$ & $2^{2.8586}$ & $1^{1.8154}$ & $3^{2.0165}$ & $5^{2.4430}$ & $5^{2.7804}$ & 1.8091 & $4^{2.0075}$ & $3^{2.4279}$ & $6^{2.7629}$ \\
\hline & $\pi / 2$ & $1^{2.6094}$ & $4^{2.9095}$ & $5^{3.5495}$ & $3^{4.0447}$ & $4^{2.5540}$ & $2^{2.8392}$ & $8^{3.4441}$ & $6^{3.9190}$ & $7^{2.5446}$ & $4^{2.8245}$ & 3.4175 & 3.8888 \\
\hline & $2 \pi / 3$ & 4.7719 & $3^{5.3307}$ & $4^{6.5235}$ & $8^{7.4307}$ & $7^{4.6635}$ & $4^{5.1883}$ & $4^{6.3019}$ & $\begin{array}{l}5^{7.1694} \\
\end{array}$ & $3^{4.6452}$ & $9^{5.1574}$ & 6.2429 & $4^{7.1033}$ \\
\hline & $3 \pi / 4$ & $3^{7.7089}$ & $2^{8.6197}$ & $7^{10.564}$ & $9^{12.031}$ & 7.5272 & $6^{8.3774}$ & $1^{10.182}$ & $6^{11.582}$ & $3^{7.4964}$ & 8.3242 & $2^{10.078}$ & $9^{11.466}$ \\
\hline \multirow{6}{*}{ 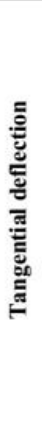 } & $\pi / 9$ & ${ }^{0.2797}$ & $9^{0.2994}$ & $9^{0.3403}$ & $6^{0.3892}$ & $06^{0.2751}$ & $99^{0.3004}$ & $92^{0.3533}$ & ${ }^{0.4009}$ & $2^{0.2743}$ & $47^{0.3023}$ & $43^{0.3609}$ & $9^{0.4083}$ \\
\hline & $\pi / 6$ & $11^{0.4330}$ & ${ }^{0.4686}$ & $27^{0.5431}$ & $32^{0.6190}$ & $31^{0.4258}$ & $75^{0.4671}$ & $1^{0.5535}$ & $62^{0.6272}$ & $15^{0.4246}$ & $7^{0.4686}$ & $65^{0.5608}$ & $42^{0.6343}$ \\
\hline & $\pi / 3$ & $3^{1.0355}$ & $6^{1.1325}$ & $9^{1.3363}$ & 1.5185 & $4^{1.0184}$ & $3^{1.1220}$ & $1^{1.3388}$ & $4^{1.5154}$ & $4^{1.0155}$ & 1.1225 & $1^{1.3464}$ & $3^{1.5222}$ \\
\hline & $\pi / 2$ & $4^{2.1803}$ & $8^{2.3919}$ & $4^{2.8372}$ & $2^{3.2210}$ & $8^{2.1445}$ & $5^{2.3656}$ & $9^{2.8285}$ & $7^{3.2006}$ & $3^{2.1385}$ & $3^{2.3647}$ & 2.8384 & $8^{3.2086}$ \\
\hline & $2 \pi / 3$ & $9^{5.2938}$ & $2^{5.8150}$ & $3^{6.9119}$ & 7.844 & $3^{5.2077}$ & $7^{5.7474}$ & $8^{6.8779}$ & $3^{7.7816}$ & $4^{5.1931}$ & $1^{5.7434}$ & $7^{6.8957}$ & 7.795 \\
\hline & $3 \pi / 4$ & $2^{9.5996}$ & 10.548 & $3^{12.544}$ & $5^{14.234}$ & $4^{9.4439}$ & $1^{10.424}$ & $1^{12.477}$ & $9^{14.115}$ & $9^{9.4175}$ & $9^{10.415}$ & $7^{12.506}$ & $4^{14.137}$ \\
\hline
\end{tabular}

Table 8. The variation of dimensionless radial and tangential displacement versus opening angle, aspect ratios and power law index for simply supported curved beam $(\mu=1)$

\begin{tabular}{|c|c|c|c|c|c|c|c|c|c|c|c|c|c|}
\hline & $\boldsymbol{\alpha}$ & & & $h=10$ & & & & $\mathrm{~h}=25$ & & & & $=75$ & \\
\hline & & $\mathrm{P}=0$ & $2^{P}=0$. & $\mathrm{P}=1$ & $P=5$ & $\mathrm{P}=0$ & $2^{P=0 .}$ & $\mathrm{P}=1$ & $P=5$ & $P=0$ & ${ }_{2}^{P=0 .}$ & $P=1$ & $P=5$ \\
\hline \multirow{6}{*}{ 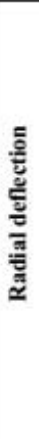 } & $\pi / 9$ & $6^{1.6284}$ & $1^{1.8077}$ & $2^{2.1892}$ & $3^{2.4972}$ & $4^{1.5973}$ & $5^{1.7724}$ & $4^{2.1436}$ & $7^{2.4403}$ & $8^{1.5920}$ & $9^{1.7660}$ & $9^{2.1346}$ & $9^{2.4294}$ \\
\hline & $\pi / 6$ & $1^{1.6812}$ & $7^{1.8674}$ & $8^{2.2639}$ & $7^{2.5820}$ & $7^{1.6488}$ & 1.8301 & $2^{2.2143}$ & $5^{2.5206}$ & $9^{1.6433}$ & $8^{1.8231}$ & $1^{2.2040}$ & $2^{2.5083}$ \\
\hline & $\pi / 3$ & $9^{2.0152}$ & $4^{2.2428}$ & $5^{2.7276}$ & $1^{3.1094}$ & $1^{1.9751}$ & $3^{2.1939}$ & $7^{2.6579}$ & $4^{3.0250}$ & $1^{1.9683}$ & $2^{2.1842}$ & $1^{2.6416}$ & $2^{3.0061}$ \\
\hline & $\pi / 2$ & $2^{2.8398}$ & $7^{3.1664}$ & 3.863 & $1^{4.4018}$ & $7^{2.7799}$ & $8^{3.0903}$ & $6^{3.7488}$ & $3^{4.2657}$ & $4^{2.7698}$ & $8^{3.0744}$ & $1^{3.7199}$ & $1^{4.2329}$ \\
\hline & $2 \pi / 3$ & $5^{5.1963}$ & $1^{5.8049}$ & $2^{7.1038}$ & $7^{8.0916}$ & $2^{5.0788}$ & $2^{5.6503}$ & $7^{6.8630}$ & $1^{7.8078}$ & $2^{5.0589}$ & 5.6168 & $8^{6.7988}$ & $4^{7.7359}$ \\
\hline & $3 \pi / 4$ & $7^{8.3970}$ & $8^{9.3891}$ & $8^{11.507}$ & $9^{13.105}$ & $7^{8.1995}$ & $8^{9.1257}$ & $6^{11.091}$ & $3^{12.617}$ & $3^{8.1661}$ & $5^{9.0678}$ & $6^{10.978}$ & $3^{12.491}$ \\
\hline \multirow{6}{*}{ 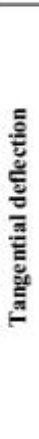 } & $\pi / 9$ & ${ }_{58}^{0.3052}$ & $\begin{array}{l}0.3266 \\
47^{2}\end{array}$ & $\begin{array}{l}0.3709 \\
49\end{array}$ & $82^{0.4242}$ & $22^{0.3001}$ & $\begin{array}{l}0.3277 \\
63\end{array}$ & $31^{0.3853}$ & $36^{0.4372}$ & ${ }_{53}^{0.2992}$ & $\begin{array}{l}0.3298 \\
07\end{array}$ & $\begin{array}{l}0.3936 \\
83\end{array}$ & $42^{0.4454}$ \\
\hline & $\pi / 6$ & $\begin{array}{l}0.4724 \\
81\end{array}$ & $29^{0.5112}$ & $54^{0.5921}$ & ${ }_{93}^{0.6749}$ & ${ }_{41}^{0.4645}$ & ${ }_{81}^{0.5095}$ & $\begin{array}{l}0.6036 \\
29\end{array}$ & $\begin{array}{l}0.6840 \\
85^{0}\end{array}$ & $96^{0.4631}$ & $42^{0.5112}$ & s9 & $13^{0.6919}$ \\
\hline & $\pi / 3$ & $2^{1.1297}$ & $1^{1.2354}$ & $6^{1.4574}$ & $8^{1.6561}$ & $5^{1.1108}$ & $8^{1.2237}$ & 1.4601 & $6^{1.6527}$ & $6^{1.1076}$ & $9^{1.2242}$ & $7^{1.4684}$ & $4^{1.6602}$ \\
\hline & $\pi / 2$ & $9^{2.3779}$ & $6^{2.6086}$ & $6^{3.0939}$ & $9^{3.5125}$ & $6^{2.3386}$ & $8^{2.5796}$ & $7^{3.0843}$ & $3^{3.4901}$ & 2.332 & $5^{2.5786}$ & $3^{3.0951}$ & $1^{3.4989}$ \\
\hline & $2 \pi / 3$ & $2^{5.7719}$ & $4^{6.3399}$ & $8^{7.5355}$ & 8.5519 & $2^{5.6775}$ & $9^{6.2658}$ & $4^{7.4982}$ & $3^{8.4834}$ & $4^{5.6615}$ & $2^{6.2614}$ & $8^{7.5176}$ & 8.4980 \\
\hline & $3 \pi / 4$ & $8^{10.464}$ & $4^{11.498}$ & $4^{13.674}$ & 15.517 & $6^{10.294}$ & $9^{11.362}$ & $8^{13.600}$ & $2^{15.387}$ & $8^{10.265}$ & 11.354 & 13.633 & $6^{15.410}$ \\
\hline
\end{tabular}

Table 9. The variation of dimensionless radial and tangential displacement versus opening angle, aspect ratios and power law index for simply supported curved beam $(\mu=2)$ 


\begin{tabular}{|c|c|c|c|c|c|c|c|c|c|c|c|c|c|}
\hline & \multirow[t]{2}{*}{$\boldsymbol{\alpha}$} & \multicolumn{4}{|c|}{$\mathrm{L} / \mathrm{h}=10$} & \multicolumn{4}{|c|}{$\mathrm{L} / \mathrm{h}=25$} & \multicolumn{4}{|c|}{$L / h=75$} \\
\hline & & $P=0$ & $2^{P=0 .}$ & $P=1$ & $P=5$ & $P=0$ & $2^{P=0 .}$ & $P=1$ & $P=5$ & $P=0$ & $2^{P=0 .}$ & $P=1$ & $P=5$ \\
\hline \multirow{6}{*}{ 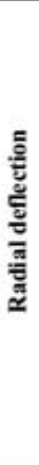 } & $\pi / 9$ & $s^{1.7592}$ & 1.9529 & $6^{2.3650}$ & $3^{2.6977}$ & $9^{1.7259}$ & $9^{1.9151}$ & $8^{2.3162}$ & $9^{2.6368}$ & $5^{1.7203}$ & $9^{1.9083}$ & $9^{2.3066}$ & $4^{2.6252}$ \\
\hline & $\pi / 6$ & $4^{1.8163}$ & $7^{2.0175}$ & $5^{2.4459}$ & $3^{2.7895}$ & $5^{1.7817}$ & $8^{1.9775}$ & $7^{2.3927}$ & $7^{2.7237}$ & $9^{1.7758}$ & $7^{1.9701}$ & $1^{2.3817}$ & $5^{2.7105}$ \\
\hline & $\pi / 3$ & $7^{2.1778}$ & $9^{2.4237}$ & $1^{2.9477}$ & $9^{3.3601}$ & $2^{2.1348}$ & $2^{2.3713}$ & $8^{2.8728}$ & $3^{3.2696}$ & $3^{2.1275}$ & 2.3609 & $9^{2.8552}$ & $9^{3.2492}$ \\
\hline & $\pi / 2$ & $4^{3.0702}$ & $9^{3.4233}$ & $4^{4.1764}$ & $9^{4.7588}$ & $1^{3.0059}$ & $4^{3.3415}$ & $3^{4.0535}$ & 4.6124 & $2^{2.9950}$ & $2^{3.3244}$ & $2^{4.0223}$ & $2^{4.5770}$ \\
\hline & $2 \pi / 3$ & 5.6208 & $\begin{array}{l}6.2790 \\
8\end{array}$ & 7.6841 & $6^{8.7525}$ & $7^{5.4940}$ & $9^{6.1122}$ & $\begin{array}{l}7.4242 \\
1\end{array}$ & $\begin{array}{l}8.4461 \\
7\end{array}$ & $1^{5.4726}$ & $1^{6.0761}$ & $6^{7.3548}$ & $5^{8.3685}$ \\
\hline & $3 \pi / 4$ & $2^{9.0852}$ & $6^{10.158}$ & $9^{12.450}$ & $9^{14.179}$ & $4^{8.8719}$ & $1^{9.8741}$ & $1^{12.001}$ & $9^{13.651}$ & $3^{8.8358}$ & 9.8115 & $9^{11.878}$ & $7^{13.515}$ \\
\hline \multirow{6}{*}{ 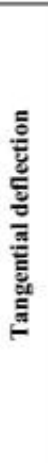 } & $\pi / 9$ & $\begin{array}{l}0.3307 \\
69^{0}\end{array}$ & $\begin{array}{l}0.3538 \\
05\end{array}$ & $\begin{array}{l}0.4015 \\
08\end{array}$ & $\begin{array}{l}0.4593 \\
04\end{array}$ & $\begin{array}{l}0.3251 \\
39\end{array}$ & $\begin{array}{l}0.3550 \\
27\end{array}$ & $\begin{array}{l}0.4172 \\
69^{-4}\end{array}$ & 0.4735 & $\begin{array}{l}0.3241 \\
86\end{array}$ & $\begin{array}{l}0.3572 \\
67\end{array}$ & $23^{0.4264}$ & 9.4824 \\
\hline & $\pi / 6$ & $\begin{array}{l}0.5119 \\
51\end{array}$ & $94^{0.5537}$ & $82^{0.6411}$ & $\begin{array}{l}0.7309 \\
53\end{array}$ & $s^{0.5032}$ & $\begin{array}{l}0.5519 \\
87\end{array}$ & 48 & $\begin{array}{l}0.7409 \\
09\end{array}$ & $\begin{array}{l}0.5017 \\
77\end{array}$ & $\begin{array}{l}0.5538 \\
06\end{array}$ & $\begin{array}{l}0.6626 \\
54\end{array}$ & $83^{0.7494}$ \\
\hline & $\pi / 3$ & 1.2239 & $6^{1.3382}$ & $3^{1.5785}$ & $5^{1.7938}$ & $7^{1.2032}$ & $3^{1.3255}$ & $9^{1.5813}$ & $8^{1.7900}$ & $8^{1.1997}$ & $9^{1.3260}$ & $4^{1.5905}$ & $5^{1.7982}$ \\
\hline & $\pi / 2$ & $4^{2.5756}$ & $3^{2.8253}$ & $8^{3.3506}$ & $6^{3.8041}$ & $4^{2.5327}$ & 2.7937 & $6^{3.3401}$ & 3.7796 & $8^{2.5254}$ & $7^{2.7925}$ & $5^{3.3518}$ & $3^{3.7891}$ \\
\hline & $2 \pi / 3$ & $4^{6.2499}$ & $5^{6.8648}$ & $3^{8.1592}$ & 9.2598 & $2^{6.1473}$ & $1^{6.7843}$ & $1^{8.1185}$ & $3^{9.1852}$ & $5^{6.1299}$ & $4^{6.7794}$ & $9^{8.1395}$ & $3^{9.2010}$ \\
\hline & $3 \pi / 4$ & 11.33 & $9^{12.448}$ & $5^{14.804}$ & $6^{16.799}$ & $2^{11.145}$ & $8^{12.301}$ & $4^{14.724}$ & $4^{16.658}$ & $9^{11.113}$ & $1^{12.292}$ & $3^{14.759}$ & $8^{16.683}$ \\
\hline
\end{tabular}

Table 10. The variation of dimensionless radial and tangential displacement versus opening angle, aspect ratios and power law index for simply supported curved beam $(\mu=3)$

\begin{tabular}{|c|c|c|c|c|c|c|c|c|c|c|c|c|c|}
\hline & $\alpha$ & & & $h=10$ & & & & $h=25$ & & & & $h=75$ & \\
\hline & & $P=0$ & $2^{\mathrm{P}=0 .}$ & $P=1$ & $P=5$ & $P=0$ & $2^{P=0 .}$ & $P=1$ & $P=5$ & $P=0$ & $2^{P=0 .}$ & $P=1$ & $P=5$ \\
\hline \multirow{6}{*}{ 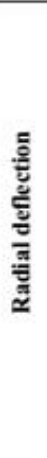 } & $\pi / 9$ & $4^{1.8900}$ & $9^{2.0980}$ & $9^{2.5408}$ & $3^{2.8982}$ & $3^{1.8546}$ & $4^{2.0579}$ & $1^{2.4889}$ & $2^{2.8334}$ & $3^{1.8486}$ & $9^{2.0506}$ & $9^{2.4786}$ & $9^{2.8209}$ \\
\hline & $\pi / 6$ & $6^{1.9514}$ & $7^{2.1676}$ & $2^{2.6279}$ & $9^{2.9969}$ & $2^{1.9146}$ & $7^{2.1250}$ & $1^{2.5712}$ & 2.9269 & $9^{1.9083}$ & $6^{2.1171}$ & 2.5594 & $8^{2.9127}$ \\
\hline & $\pi / 3$ & $5^{2.3404}$ & $4^{2.6047}$ & $8^{3.1677}$ & $8^{3.6109}$ & $2^{2.2945}$ & $2^{2.5487}$ & 3.0878 & $1^{3.5142}$ & $4^{2.2867}$ & $8^{2.5375}$ & $7^{3.0689}$ & $5^{3.4924}$ \\
\hline & $\pi / 2$ & $5^{3.3006}$ & $2^{3.6803}$ & $9^{4.4898}$ & $7^{5.1159}$ & $4^{3.2318}$ & 3.5927 & $1^{4.3582}$ & $7^{4.9590}$ & $9^{3.2201}$ & $6^{3.5743}$ & $3^{4.3247}$ & $3^{4.9211}$ \\
\hline & $2 \pi / 3$ & $5^{6.0452}$ & $5^{6.7532}$ & $8^{8.2643}$ & $4^{9.4134}$ & $2^{5.9093}$ & $7^{6.5742}$ & $4^{7.9853}$ & $4^{9.0845}$ & 5.8863 & $2^{6.5354}$ & $3^{7.9108}$ & $5^{9.0011}$ \\
\hline & $3 \pi / 4$ & $6^{9.7733}$ & $1^{10.928}$ & 13.394 & $8^{15.253}$ & $1^{9.5443}$ & $4^{10.622}$ & $7^{12.910}$ & $5^{14.686}$ & $3^{9.5055}$ & $2^{10.555}$ & $3^{12.779}$ & $1^{14.540}$ \\
\hline \multirow{6}{*}{ 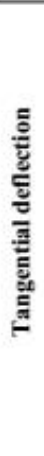 } & $\pi / 9$ & $8^{0.3562}$ & $62^{0.3809}$ & $\begin{array}{l}0.4320 \\
67\end{array}$ & 27 & $\begin{array}{l}0.3501 \\
56\end{array}$ & $\begin{array}{l}0.3822 \\
91\end{array}$ & 08 & $6^{0.5097}$ & $19^{0.3491}$ & $27^{0.3847}$ & 64 & $\begin{array}{l}0.5195 \\
46\end{array}$ \\
\hline & $\pi / 6$ & $21^{0.5514}$ & $6^{0.5963}$ & $1^{0.6902}$ & $\begin{array}{l}0.7869 \\
14\end{array}$ & $6^{0.5419}$ & ${ }_{93}^{0.5943}$ & $\begin{array}{l}0.7038 \\
68\end{array}$ & $33^{0.7977}$ & $\begin{array}{l}0.5403 \\
58\end{array}$ & 69 & $49^{0.7135}$ & $53^{0.8070}$ \\
\hline & $\pi / 3$ & $9^{1.3180}$ & $2^{1.4411}$ & 1.6996 & $2^{1.9315}$ & $9^{1.2956}$ & $8^{1.4272}$ & $8^{1.7026}$ & 1.9274 & $9^{1.2918}$ & $9^{1.4278}$ & 1.7126 & $5^{1.9362}$ \\
\hline & $\pi / 2$ & $9^{2.7732}$ & $1^{3.0420}$ & 3.6074 & $3^{4.0957}$ & $2^{2.7268}$ & $2^{3.0077}$ & $4^{3.5959}$ & $6^{4.0690}$ & $6^{2.7189}$ & $9^{3.0064}$ & $8^{3.6085}$ & $6^{4.0793}$ \\
\hline & $2 \pi / 3$ & $7^{6.7279}$ & $7^{7.3897}$ & $8^{8.7828}$ & $9^{9.9676}$ & $2^{6.6171}$ & $3^{7.3027}$ & $8^{8.7387}$ & $4^{9.8870}$ & $5^{6.5983}$ & $5^{7.2974}$ & $9^{8.7614}$ & $4^{9.9040}$ \\
\hline & $3 \pi / 4$ & $2^{12.195}$ & $4^{13.399}$ & $6^{15.934}$ & $1^{18.082}$ & $9^{11.995}$ & $6^{13.240}$ & 15.848 & $7^{17.929}$ & $1^{11.962}$ & $1^{13.230}$ & $6^{15.885}$ & 17.957 \\
\hline
\end{tabular}

Table 11. The variation of dimensionless radial and tangential displacement versus opening angle, aspect ratios and power law index for simply supported curved beam $(\mu=4)$

The variation of radial displacement versus the power law index for different aspect ratios and for opening angles of 
$\frac{3 \pi}{4}, \frac{2 \pi}{3}, \frac{\pi}{2}, \frac{\pi}{3}, \frac{\pi}{6}, \frac{\pi}{9}$. the radial displacement in high aspect ratios is considerable which increases as the power law index increases. It should be noted that in the vibration analysis increasing the aspect ratio results in increasing the frequencies while in bending analysis increasing the mentioned values tends to decrease the radial displacement.
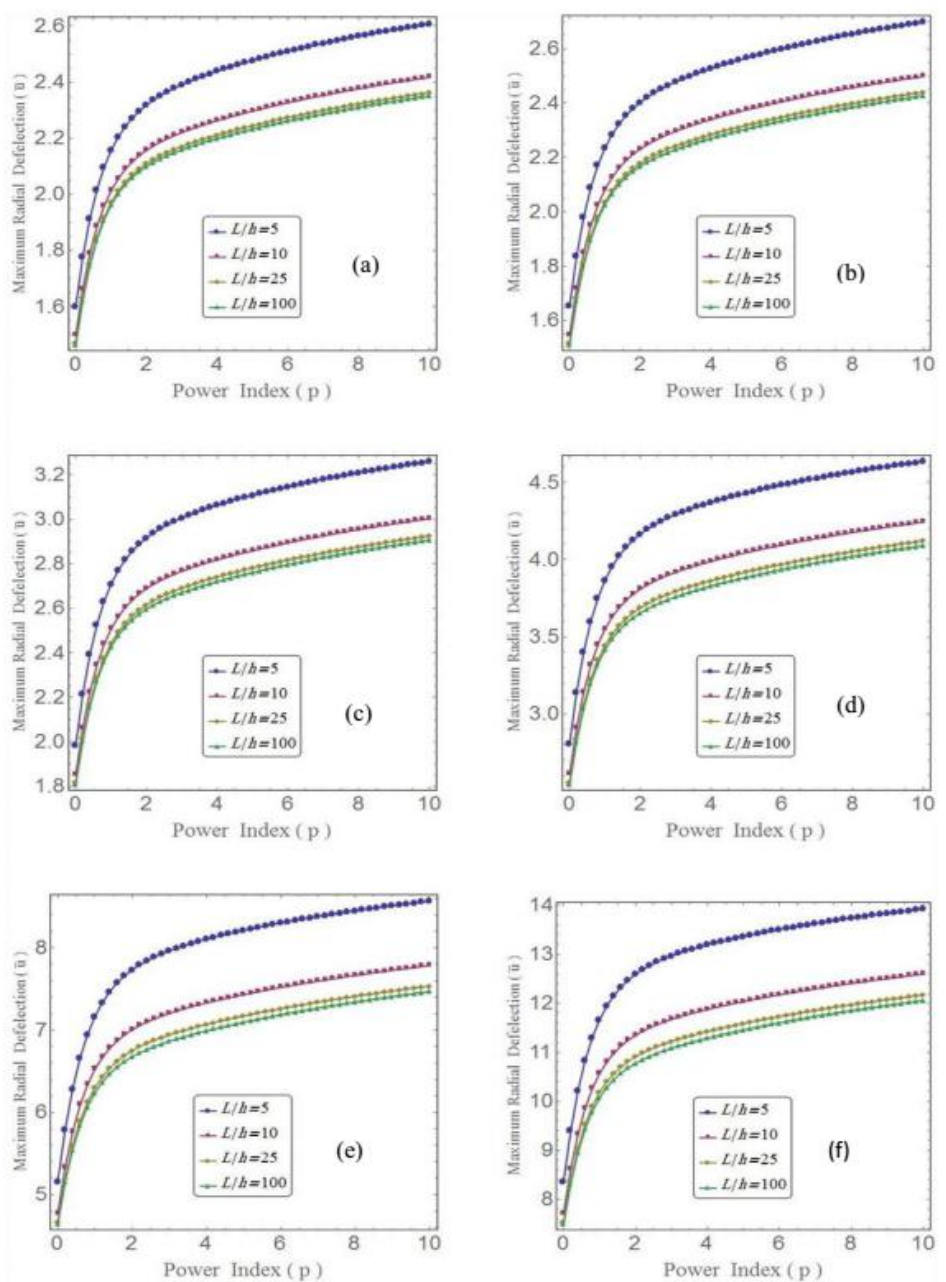

Figure4. The variation of dimensionless radial displacement versuspower law indexand for selectedaspect ratios and for opening angle simply supported curved beam $(\mu=4)$

\section{Conclusion}

In this work, the nonlocal Timoshenko beam model of a curved beam is used to analyze the bending and vibration problem. Using the Hamilton principle and the nonlocal theory the differential equations and governing equations for a curved FG nanobeam were obtained respectively. The Navier method was employed to propose a vibration and bending solution. The influence of some parameters such as curved FG nanobeam opening angle, nonlocal parameter, mode number, aspect ratio and the influence of power index law of FGM on the non-dimensionalized frequency, non-dimensionalized radial displacement and non-dimensionalized tangential displacement were thoroughly studied in this work by employing several tables and graphs. The results revealed that increasing the aspect ratio results in increasing and decreasing the non-dimensionalized frequency and displacements respectively. As the nonlocal parameter increases the amount of frequency and the radial and tangential displacement decreases and increases respectively. It was also concluded that the non-dimensionalized frequency and the radial and tangential displacement decreases and increases with increasing the opening angle respectively which shows the importance of the angle parameter. 


\section{References}

1. Kiani, K. Small-scale effect on the vibration of thin nanoplates subjected to a moving nanoparticle via nonlocal continuum theory. Journal of Sound and Vibration 2011; 330(20): 4896-4914.

2. Niknam, H. and M. Aghdam. A semi analytical approach for large amplitude free vibration and buckling of nonlocal FG beams resting on elastic foundation. Composite Structures 2015; 119: 452-462.

3. Eringen, A.C. and D. Edelen. On nonlocal elasticity. International Journal of Engineering Science 1972; 10(3): 233-248.

4. Zhang, Y., G. Liu, and X. Xie. Free transverse vibrations of double-walled carbon nanotubes using a theory of nonlocal elasticity. Physical Review B 2005; 71(19): 195404.

5. Wang, Q. and V. Varadan. Vibration of carbon nanotubes studied using nonlocal continuum mechanics. Smart Materials and Structures 2006; 15(2): 659.

6. Hu, Y.-G., et al. Nonlocal shell model for elastic wave propagation in single-and double-walled carbon nanotubes. Journal of the Mechanics and Physics of Solids 2008. 56(12): p. 3475-3485.

7. Reddy, J. and S. Pang. Nonlocal continuum theories of beams for the analysis of carbon nanotubes. Journal of Applied Physics 2008; 103(2): 023511.

8. Aydogdu, M. A general nonlocal beam theory: its application to nanobeam bending, buckling and vibration. Physica E: Low-dimensional Systems and Nanostructures 2009; 41(9): 1651-1655.

9. Reddy, J. Nonlocal nonlinear formulations for bending of classical and shear deformation theories of beams and plates. International Journal of Engineering Science 2010; 48(11): 1507-1518.

10. Hosseini-Hashemi, S., et al. Surface effects on free vibration of piezoelectric functionally graded nanobeams using nonlocal elasticity. Acta Mechanica 2014; 225(6): 1555-1564.

11. Pirmohammadi, A.A., et al. Modeling and active vibration suppression of a single-walled carbon nanotube subjected to a moving harmonic load based on a nonlocal elasticity theory. Applied Physics A 2014; 117(3): 1547-1555.

12. Shi, J.-X., et al. Nonlocal vibration analysis of nanomechanical systems resonators using circular double-layer graphene sheets. Applied Physics A 2014; 115(1): 213-219.

13. Ansari, R., R. Gholami, and S. Sahmani. Prediction of compressive post-buckling behavior of single-walled carbon nanotubes in thermal environments. Applied Physics A 2013; 113(1): 145-153.

14. Reddy, J. Nonlocal theories for bending, buckling and vibration of beams. International Journal of Engineering Science 2007; 45(2): 288-307.

15. Rahmani, O. and A.A. Jandaghian. Buckling analysis of functionally graded nanobeams based on a nonlocal third-order shear deformation theory. Applied Physics A 2015; 119(3): 1019-1032.

16. Natsuki, T., N. Matsuyama, and Q.-Q. Ni. Vibration analysis of carbon nanotube-based resonator using nonlocal elasticity theory. Applied Physics A 2015; 120(4): 1309-1313.

17. Rahmani, O., et al. Torsional Vibration of Cracked Nanobeam Based on Nonlocal Stress Theory with Various Boundary Conditions: An Analytical Study. International Journal of Applied Mechanics 2015; 07(03): 1550036.

18. Hosseini, S. and O. Rahmani. Exact solution for axial and transverse dynamic response of functionally graded nanobeam under moving constant load based on nonlocal elasticity theory. Meccanica 2017. 52(6): 1441-1457.

19. Hosseini, S.A.H. and O. Rahmani. Free vibration of shallow and deep curved FG nanobeam via nonlocal Timoshenko curved beam model. Applied Physics A 2016; 122(3): 1-11.

20. Misagh, Z. and H. Seyed Amirhosein. A semi analytical method for electro-thermo-mechanical nonlinear vibration analysis of nanobeam resting on the Winkler-Pasternak foundations with general elastic boundary conditions. Smart Materials and Structures 2016; 25(8): 085005.

21. Sourki, R. and S.A. Hosseini. Coupling effects of nonlocal and modified couple stress theories incorporating surface energy on analytical transverse vibration of a weakened nanobeam. The European Physical Journal Plus 2017; 132(4): 184.

22. Lee, H.-L. and W.-J. Chang. Surface effects on frequency analysis of nanotubes using nonlocal Timoshenko beam theory. Journal of Applied Physics 2010; 108(9): 093503.

23. Wang, C., Y. Zhang, and X. He. Vibration of nonlocal Timoshenko beams. Nanotechnology 2007; 18(10): 105401.

24. Rahmani, O. and O. Pedram. Analysis and modeling the size effect on vibration of functionally graded nanobeams based on nonlocal Timoshenko beam theory. International Journal of Engineering Science 2014; 77: 55-70.

25. Ebrahimi, F. and E. Salari. Nonlocal thermo-mechanical vibration analysis of functionally graded nanobeams in thermal environment. Acta Astronautica 2015; 113: 29-50.

26. Komijani, M., et al. Vibration of thermo-electrically post-buckled rectangular functionally graded piezoelectric beams. Composite Structures 2013; 98: 143-152.

27. Eltaher, M., S.A. Emam, and F. Mahmoud. Static and stability analysis of nonlocal functionally graded nanobeams. Composite Structures 2013; 96: 82-88. 
28. Eltaher, M., et al. Static and buckling analysis of functionally graded Timoshenko nanobeams. Applied Mathematics and Computation 2014; 229: 283-295.

29. Şimşek, M. and H. Yurtcu. Analytical solutions for bending and buckling of functionally graded nanobeams based on the nonlocal Timoshenko beam theory. Composite Structures 2013; 97: 378-386.

30. KANANIPOUR, H, I. KERMANI, and H. CHAVOSHI. Nonlocal beam model for dynamic analysis of curved nanobeams and rings.

31. Farshi, B., A. Assadi, and A. Alinia-Ziazi. Frequency analysis of nanotubes with consideration of surface effects. Applied Physics Letters 2010; 96: 093105.

32. Wang, C.M. and W. Duan. Free vibration of nanorings/arches based on nonlocal elasticity. Journal of Applied Physics 2008; 104: 014303.

33. Medina, L., R. Gilat, and S. Krylov. Symmetry breaking in an initially curved micro beam loaded by a distributed electrostatic force. International Journal of Solids and Structures 2012; 49: 1864-1876. 